\title{
Mathematical formulations for elastic magneto-electrically coupled soft materials at finite strains: Time-independent processes
}

\author{
R. Bustamante ${ }^{1}$, M.H.B.M. Shariff' ${ }^{2}$, M. Hossain ${ }^{3}$ \\ ${ }^{1}$ Departamento de Ingeniería Mecánica, Universidad de Chile \\ Beauchef 851, Santiago Centro, Santiago, Chile. \\ ${ }^{2}$ Department of Mathematics \\ Khalifa University of Science and Technology, United Arab Emirates. \\ ${ }^{3}$ Zienkiewicz Centre for Computational Engineering, College of Engineering \\ Swansea University, Swansea, United Kingdom.
}

November 18, 2020

\begin{abstract}
Recently, among other smart and multifunctional materials, magneto-electric soft materials are expected to open a new horizon with myriad of potential applications such as wireless energy harvesting, spintronics and nonvolatile memories, magneto-electric random access memory, to mention a few. Magneto-electric coupling can be defined as the ability of a material to electrically polarize upon the application of a magnetic field and conversely, to magnetize under the application of an electric field. In contrast to traditional multi-ferroic hard materials, magneto-electric soft materials are of largely deformable where electric and magnetic fields and mechanical deformations are intricately coupled at finite strians. In this contribution, we will emphasis to formulate generalised mathematical frameworks of finitely deformed magneto-electric soft materials. After elaborating fundamental and governing equations, some homogeneous and non-homogeneous classical boundary value problems are studied under magneto-electrically coupled loads.
\end{abstract}

\section{Introduction}

In recent years, smart and functional materials have attracted considerable attention among cross-boundary research communities due to their wide range of potential applications. These fast growing innovative materials can change their size, shape, stiffness, and other mechanical properties upon the application of external fields (temperature, $\mathrm{pH}$, light, electric and or magnetic field). Among other functional materials, magneto-active polymers (MAPs) and electro-active polymers (EAPs) are promising candidates for use in various engineering applications, for example, as mini- or micro-scale soft, biodegradable, and flexible robots in targeted drug delivery, stretch sensors in wearable devices and in structural health monitoring systems, actuators in artificial muscles, biomedical devices, biomimetic systems, and 
energy harvesters generating power by using ambient motions [43, 42, 49, 22]. An external electric field or electric potential difference in EAPs can result in extremely large deformations in contrast to traditional piezoelectric materials that have only small-scale actuations $[1,8,9,4,5,6,7,40,41,42,44,77,62,63]$. In the case of MAPs, a remotely applied magnetic field can deform soft materials filled with magnetites (iron oxide particles). While EAPs can either be pure polymers or polymers filled with high permittivity fillers, in MAPs, micron-size iron particles are mixed to prepare polymeric composites that are responsive to external magnetic fields. For an exhaustive review on experimental study of MAPs, see Bastola and Hossain [3]. In contrast to EAPs, MAPs are normally actuated remotely without any physical attachment of the external field to the surface of an actuated body $[21,23,32,36,38,44,52,53,54,50,77,75]$.

In comparison to research efforts in MAPs and EAPs separately, interests in smart materials, where a combined magneto-electrically (ME) coupling occurs, have increased significantly in the last decade. Most of the earlier works in ME are in the areas of multi-ferroic hard materials (i.e., ceramics and metals) that have small field-responsive deformations. Thanks to some high-tech applications of ME materials such as the design of magneto-electric random access memories (MERAM), a significant amount of research efforts as well as device making processes have been achieved in ME for hard materials, see for example [29, 31, 34, 37, 72, 64, 76]. In the case of MERAMs, a short electric signal causes an enduring change of magnetization that is a very desirable property. In contrast to the traditional magnetic RAM (random access memory) technology, the new class of storage devices made of MERAM would enable electric-write magnetic-read memories which outperform the former one [2]. Further applications of ME coupled material is the measurement of magnetic fields and also electric current, particularly in the low-frequency range as, for instance, documented by Fetisov et al. [33]. For an overview of the vast amount of research and possible applications in this wide field, see for example, Nan et al. [56], Pyatakov et al. [59].

Although magneto-electric materials were discovered in the middle of the last century, in contrast to small-strained hard ME materials, an interesting finite strain alternative has been very recently proposed by Liu and Sharma [47], Krichen et al. [46]. This new class of smart materials has been appeared exploiting the strain-mediated magneto-electric coupling in soft composites. ME coupled materials mimic the concepts from multi-ferroics hard-matter composites. The basic idea behind this approach is to combine the magneto- and electromechanical responses of composites consisting of a soft matrix carrying magnetic inclusions. Magneto-electric coupling in a soft material can be explained in the following way. A soft deformable dielectric thin film coated with two compliant electrodes under a potential difference will deform and polarise thanks to an electric Maxwell stress. If the compressed thin film with the pre-existing electric field is exposed to an external magnetic, it will further deform due to the magnetic Maxwell stress and the film will polarise additionally. The latter form of deformation will alter the pre-existing electric field and such a change in the electric field can be measured as current. Note that such a mechanism does not require creating complex composites (neither piezoelectric nor magnetostrictive) and can be universally employed for all soft materials. Despite that such composites are straightforward to manufacture and have cheap constituents, they did not gain much attention up to now. Rambausek and Keip [60] demonstrate that ME coupling induced by finite deformations could be of significant magnitude. Furthermore, Liu and Sharma [47], Liu [48], Krichen et al. [46] highlighted that the 
ME coupling effect through soft-matter composites is a plausible candidate mechanism for magneto-reception in certain biological contexts.

The mathematical foundations of the coupling of electromagnetic fields in finite strains are well documented in some earlier publications, see for example the works of Pao and NematNasser [58], Eringen and Maugin [30], Maugin [51], Brown [11], Monk [55], Kovetz [45] and the references therein. In a series of papers and monographs, Dorfmann and Ogden developed constitutive frameworks for the coupling of magnetic, electric and mechanical fields which are based on the so-called total energy [10, 25, 26, 27, 28, 24]. Their modelling frameworks mainly assume isotropy of the polymeric composites. It has been shown that the total stress tensor and the magnetic field can be expressed as simple derivatives of the total energy function with respect to the deformation gradient and the magnetic induction. They presented analytical solutions of some classical non-homogeneous boundary value problems in which it has been shown that any of the magnetic variables, i.e., the magnetic induction vector, the magnetic field vector or the magnetization vector can be used as an independent variable in the problem formulation. A significant amount of contributions on the modelling of magneto-mechanically coupled problems were published by Bustamante and coworkers $[13,20,12,15,16]$ extending the work of Dorfmann et al. [25] by a constitutive model for transversely isotropic MAPs. In contrast to the Dorfmann, Odgen and Bustamante's constitutive frameworks expressed in terms of the classical invariants of Rivlin and Spencer [73], recently Shariff et al. $[66,67,68,69,71]$ reformulated constitutive models of MAPs and EAPs that are based on a class of spectral invariants.

Regarding the nature of the constitutive equations proposed in the works mentioned above, the main assumption has been to expresses the stress and one of the electric or magnetic variables, in terms of the strains and the other (independent) electromagnetic variables, but recently some new types of implicit relations have been proposed for electro- and magnetoelastic bodies, wherein the stresses, strains and electromagnetic variables are obtained from an implicit tensor relations and an implicit vector relation, see [17, 18, 14].

Mathematical frameworks and constitutive modelling of magneto-electro-mechanically coupled soft materials at finite strain are limited in the literature. Santapuri et al. [61] develop thermodynamically consistent fully-coupled finite deformation thermo-electro-magnetomechanics of multifunctional materials. In that article, they develop a comprehensive catalogue of free energies, state variables, and state equations. Liu [48] presents an energy formulation of continuum magneto-electro-elasticity based on the principle of minimum free energy, where he proposes a form of total free energy of the system in three dimensions, and then systematically derive the theories for a hierarchy of materials including dielectric elastomers, piezoelectric ceramics, ferro-electrics, flexoelectric materials, magnetic elastomers, magneto-electric materials, piezo-electric-magnetic materials among others. Syfris et al. [65] constitutively characterize electro-magneto-mechanical interactions of graphene at continuum level using the classical theory of invariants for the specific class of graphene's symmetry. In a very recent contribution, Rambausek and Keip [60] demonstrate that ME coupling induced by finite deformations could be of significant magnitude in soft-matter-based magnetic and electric composites. They realise shape effects as a special non-local phenomenon in magnetoand electro-elasticity. 
The aims of the current contribution are two-folds. First, we present a comprehensive mathematical framework illustrating the relevant and fundamental equations that present magnetoelectro-mechanical coupling at finite strains, under the assumption that the stresses are functions of the strains and one of the electric and magnetic variables (independent variables), and that the other electric and magnetic variables are also functions of the strains and the independent electric and magnetic variables. An important ingredient of our formulation is the use of the theory of spectral invariants of Shariff for the energy function in the case of a transversely isotropic body [66, 67, 68, 69, 71]. Another contribution of this article is to present in detail the results for some few boundary value problems considering homogeneous and non-homogeneous deformations, for the above constitutive equations, with particular relevance to problems that can be used in the future in experimental research to obtain expressions for the constitutive equations.

In Section 2 we present a brief summary of the most important equations of the theory of electromagnetic interactions with elastic solids. In Section 3 spectral invariants are formulated for the total energy function in the case of a transversely isotropic magneto-electro-elastic body, and constitutive equations are obtained for the total stress, the magnetic field and the electric displacement. In Section 4 some boundary value problems are studied, namely, the uniform extension/compression of a cylinder, the uniform extension of a thin slab, the biaxial deformation of a thin plate, the shear of a slab and the non-uniform deformation of a cylindrical annulus under inflation and extension. In Section 5 a simplified expression for the total energy function is proposed, and the behaviour of a cylinder in tension is studied. In Section 6 some final remarks are given.

\section{Basic equations}

\subsection{Kinematics and balance of mass}

Let $X \in \mathscr{B}$ denote a point in a body $\mathscr{B}$, the position of that point in the reference configuration $\mathscr{B}_{r}$ is denoted as $\mathbf{X}$, while the position in the current configuration $\mathscr{B}_{t}$ is denoted $\mathbf{x}$. It is assumed that there exists a one-to-one function $\chi$ such that $\mathbf{x}=\chi(\mathbf{X}, t)$, where $t$ is time. The deformation gradient, the left and right Cauchy Green tensors, and $J$, respectively, are defined as

$$
\mathbf{F}=\frac{\partial \mathbf{x}}{\partial \mathbf{X}}, \quad \mathbf{B}=\mathbf{F F}^{\mathrm{T}}, \quad \mathbf{C}=\mathbf{F}^{\mathrm{T}} \mathbf{F}, \quad J=\operatorname{det} \mathbf{F},
$$

where it is assumed that $J>0$ always.

The balance of mass can be stated as $\dot{\rho}+\operatorname{div}(\rho \mathbf{v})=0$, where $\rho$ is the density of the body and $\mathbf{v}=\dot{\mathbf{x}}$ is the velocity in the current configuration. From now on it is assumed that $\mathbf{v}=\mathbf{0}$. In this article repetition of index does not mean summation in that index, and all index go from 1 to 3 unless it is stated otherwise. More details about kinematics see, for example, $[74,57]$.

\section{$2.2 \quad$ Field equations for electro-magneto active bodies}

In this section, we review briefly the basic concept and equations for continua media interacting with electromagnetic fields. Details about the different equations and relations presented in this section can be found, for example, in [30, 39, 51, 55, 58]. 


\subsubsection{Maxwell equations}

The Maxwell equations in the case there is no time dependence ${ }^{1}$ are:

$$
\operatorname{div} \mathbb{B}=0, \quad \operatorname{curl} \mathbb{E}=\mathbf{0}, \quad \operatorname{div} \mathbb{D}=0, \quad \operatorname{curl} \mathbb{H}=\mathbf{0},
$$

where $\mathbb{B}$ is the magnetic induction, $\mathbb{E}$ is the electric field, $\mathbb{D}$ is the electric displacement, and $\mathbb{H}$ is the magnetic field. For a vector field $\mathbb{A}$, the equations $\operatorname{curl} \mathbb{A}=\mathbf{0}$ and $\operatorname{div} \mathbb{A}=0$ in cylindrical coordinates $(r, \theta, z)$ become:

$$
\frac{1}{r} \frac{\partial \mathbb{A}_{z}}{\partial \theta}-\frac{\partial \mathbb{A}_{\theta}}{\partial z}=0, \quad \frac{\partial \mathbb{A}_{r}}{\partial z}-\frac{\partial \mathbb{A}_{z}}{\partial r}=0, \quad \frac{\partial}{\partial r}\left(r \mathbb{A}_{\theta}\right)-\frac{\partial \mathbb{A}_{r}}{\partial \theta}=0,
$$

and

$$
\frac{1}{r} \frac{\partial}{\partial r}\left(r \mathbb{A}_{r}\right)+\frac{1}{r} \frac{\partial \mathbb{A}_{\theta}}{\partial \theta}+\frac{\partial \mathbb{A}_{z}}{\partial z}=0
$$

respectively.

For vacuum the following relations between the electric variables and the magnetic variables are valid

$$
\mathbb{D}=\varepsilon_{o} \mathbb{E}, \quad \mathbb{B}=\mu_{o} \mathbb{H},
$$

where $\varepsilon_{o}$ is the electric permittivity and $\mu_{o}$ is the magnetic permeability in vacuum, respectively. For condensed matter, two extra fields are necessary, which are the electric polarization $\mathbb{P}$ and the magnetization $\mathbb{M}$ that are defined as

$$
\mathbb{D}=\varepsilon_{o} \mathbb{E}+\mathbb{P}, \quad \mathbb{B}=\mu_{o}(\mathbb{H}+\mathbb{M}) .
$$

\subsubsection{Equation of equilibrium}

The equation of equilibrium is

$$
\operatorname{div} \boldsymbol{\sigma}+\mathbf{f}_{\mathrm{e}}+\mathbf{f}=\mathbf{0},
$$

where $\boldsymbol{\sigma}$ is the Cauchy stress tensor, $\mathbf{f}_{\mathrm{e}}$ corresponds to the body forces caused by the electromagnetic interactions, and $\mathbf{f}$ represents the body forces that are not caused by electromagnetic fields. The vector $\mathbf{f}_{\mathrm{e}}$ is given as $\mathbf{f}_{\mathrm{e}}=(\operatorname{grad} \mathbb{E}) \mathbb{P}+(\operatorname{grad} \mathbb{E}) \mathbf{M}$. The balance of angular momentum gives

$$
\epsilon_{i j k} \sigma_{j k}=-\mathbb{L}_{i},
$$

where $\epsilon_{i j k}$ is the permutation symbol and $\mathbb{L}_{i}$ are the components of the vector field $\mathbb{L}=$ $\mathbb{P} \times \mathbb{E}+\mathbb{M} \times \mathbb{B}$. It is possible to see that $\boldsymbol{\sigma}$ is in general a non-symmetric second order tensor. The following identities hold (see, for example, $[25,26,27,28,24])(\operatorname{grad} \mathbb{E})^{\mathrm{T}} \mathbb{P}=$ $\operatorname{div}\left[\mathbb{D} \otimes \mathbb{E}-\frac{1}{2} \varepsilon_{o}(\mathbb{E} \cdot \mathbb{E}) \mathbf{I}\right],(\operatorname{grad} \mathbb{B})^{\mathrm{T}} \mathbf{M}=\operatorname{div}\left\{\frac{1}{\mu_{o}}\left[\mathbb{B} \otimes \mathbb{B}-\frac{1}{2}(\mathbb{B} \cdot \mathbb{B}) \mathbf{I}\right]+(\mathbf{M} \cdot \mathbb{B}) \mathbf{I}-\mathbb{B} \otimes \mathbf{M}\right\}$, from where the 'total stress' tensor $\boldsymbol{\tau}$ can be defined as

$$
\boldsymbol{\tau}=\boldsymbol{\sigma}+\mathbb{D} \otimes \mathbb{E}-\frac{1}{2} \varepsilon_{o}(\mathbb{E} \cdot \mathbb{E}) \mathbf{I}+\frac{1}{\mu_{o}}\left[\mathbb{B} \otimes \mathbb{B}-\frac{1}{2}(\mathbb{B} \cdot \mathbb{B}) \mathbf{I}\right]+(\mathbb{M} \cdot \mathbb{B}) \mathbf{I}-\mathbb{B} \otimes \mathbf{M},
$$

where $\mathbf{I}$ is the second order identity tensor. Using this in (8) we obtain $\boldsymbol{\tau}^{\mathrm{T}}=\boldsymbol{\tau}$. On the other hand using (9) in (7) we have

$$
\operatorname{div} \boldsymbol{\tau}+\mathbf{f}=\mathbf{0} .
$$

\footnotetext{
${ }^{1}$ As well as this, it is assumed that the volumetric electric current $\mathbb{J}$ and the volumetric distribution of electric charges in the body $\mathbf{q}_{\mathrm{e}}$ are zero, i.e., $\boldsymbol{J}=\mathbf{0}, \mathbf{q}_{\mathrm{e}}=0$.
} 


\subsection{Continuity conditions}

For a body that can react to electromagnetic fields, the boundary conditions are not trivial, since the Maxwell equations (2) must be satisfied for the body, and also for the exterior surrounding space, which for simplicity we assume is vacuum. Considering the notation $\llbracket a \rrbracket=a^{(\mathrm{o})}-a^{(\mathrm{i})}$, where (o) means outside the body and (i) means inside the body (in both cases very close to the surface of the body), for the electromagnetic variables we have the following continuity conditions (see, for example, [30, 51]):

$$
\mathbf{n} \cdot \llbracket \mathbb{B} \rrbracket=0, \quad \mathbf{n} \times \llbracket \mathbb{E} \rrbracket=\mathbf{0}, \quad \mathbf{n} \cdot \llbracket \mathbb{D} \rrbracket=0, \quad \mathbf{n} \times \llbracket \mathbb{H} \rrbracket=\mathbf{0},
$$

where $\mathbf{n}$ is the outward unitary normal vector to the surface of the body, and it is assumed that there is no surface free electric charges and electric current, recalling also that $\mathbf{v}=\mathbf{0}$. In the case of the Cauchy stress tensor $\boldsymbol{\sigma}$, it should satisfy the continuity condition $\llbracket \boldsymbol{\sigma}-\boldsymbol{\sigma}_{\mathrm{M}} \rrbracket \mathbf{n}=\mathbf{0}$, where $\boldsymbol{\sigma}_{\mathrm{M}}$ is called the Maxwell stress tensor and is given by $\boldsymbol{\sigma}_{\mathrm{M}}=\frac{1}{2}\left(\varepsilon_{o} \mathbb{E} \cdot \mathbb{E}+\frac{1}{\mu_{o}} \mathbb{B} \cdot \mathbb{B}-2 \mathbb{M} \cdot \mathbb{B}\right) \mathbf{I}-\left(\varepsilon_{o} \mathbb{E} \otimes \mathbb{E}+\mathbb{P} \otimes \mathbb{E}+\frac{1}{\mu_{o}} \mathbb{B} \otimes \mathbb{B}-\mathbb{B} \otimes \mathbf{M}\right) . \quad$ Using (9) in the above continuity condition we obtain

$$
\llbracket \tau \rrbracket \mathbf{n}=\mathbf{0} .
$$

The above equation is equivalent to $\left(\boldsymbol{\tau}^{(\mathrm{o})}-\boldsymbol{\tau}^{(\mathrm{i})}\right) \mathbf{n}=\mathbf{0}$, where $\boldsymbol{\tau}^{(\mathrm{i})}$ can be identified simply as $\tau$ the total stress tensor inside the body and

$$
\boldsymbol{\tau}^{(\mathrm{o})}=\boldsymbol{\tau}_{\mathrm{M}}=\varepsilon_{o}\left[\mathbb{E}^{(\mathrm{o})} \otimes \mathbb{E}^{(\mathrm{o})}-\frac{1}{2}\left(\mathbb{E}^{(\mathrm{o})} \cdot \mathbb{E}^{(\mathrm{o})}\right) \mathbf{I}\right]+\frac{1}{\mu_{o}}\left[\mathbb{B}^{(\mathrm{o})} \otimes \mathbb{B}^{(\mathrm{o})}-\frac{1}{2}\left(\mathbb{B}^{(\mathrm{o})} \cdot \mathbb{B}^{(\mathrm{o})}\right) \mathbf{I}\right],
$$

where $\boldsymbol{\tau}_{M}$ is the total Maxwell stress outside the body where $\mathbb{P}^{(0)}=\mathbf{0}, \mathbf{M}^{(0)}=\mathbf{0}$ in vacuum.

\subsection{Constitutive equations}

We revisit some basic equations and relations for continuum media interacting with electromagnetic fields, by presenting constitutive equations that are derived from the first and second laws of thermodynamics. In terms of the Helmholtz function $\psi=\psi(\mathbf{F}, \mathbb{E}, \mathbb{B}, \theta)$ the following constitutive equations are found (see details in $[30,51]$ )

$$
\mathbf{F}^{-1} \boldsymbol{\sigma}=\rho \frac{\partial \psi}{\partial \mathbf{F}}, \quad \mathbb{P}=-\rho \frac{\partial \psi}{\partial \mathbb{E}}, \quad \mathbf{M}=-\rho \frac{\partial \psi}{\partial \mathbb{B}}, \quad s=-\frac{\partial \psi}{\partial \theta},
$$

where $s$ and $\theta$ are the entropy and the absolute temperature, respectively. From now on it is assumed that $\theta$ is constant.

The Lagrangian magnetic induction, magnetic field, electric field, and the electric displacement, are defined as (see, for example, $[25,26,27,28,24]$ )

$$
\mathbb{B}_{\mathrm{L}}=J \mathbf{F}^{-1} \mathbb{B}, \quad \mathbb{H}_{\mathrm{L}}=\mathbf{F}^{\mathrm{T}} \mathbb{H}, \quad \mathbb{E}_{\mathrm{L}}=\mathbf{F}^{\mathrm{T}} \mathbb{E}, \quad \mathbb{D}_{\mathrm{L}}=J \mathbf{F}^{-1} \mathbb{D} .
$$

respectively. Let us express $\psi$ in terms of a function $\Phi$ as $\psi(\mathbf{F}, \mathbb{E}, \mathbb{B})=\Phi\left(\mathbf{F}, \mathbb{E}_{\mathrm{L}}, \mathbb{B}_{\mathrm{L}}\right)$, then from (14) 1 we obtain $\boldsymbol{\sigma}=\rho \mathbf{F} \frac{\partial \psi}{\partial \mathbf{F}}=\rho \mathbf{F} \frac{\partial \Phi}{\partial \mathbf{F}}-(\mathbf{M} \cdot \mathbb{B}) \mathbf{I}+\mathbb{B} \otimes \mathbf{M}-\mathbb{D} \otimes \mathbb{E}$, and from (9) we have $\boldsymbol{\tau}=$ $\rho \mathbf{F} \frac{\partial \Phi}{\partial \mathbf{F}}+\frac{1}{\mu_{o}}\left[\mathbb{B} \otimes \mathbb{B}-\frac{1}{2}(\mathbb{B} \cdot \mathbb{B}) \mathbf{I}\right]+\varepsilon_{o}\left[\mathbb{E} \otimes \mathbb{E}-\frac{1}{2}(\mathbb{E} \cdot \mathbb{E}) \mathbf{I}\right]$. Let us define the total nominal stress tensor $\mathbf{T}$ as $\mathbf{T}=J \mathbf{F}^{-1} \boldsymbol{\tau}=\rho_{0} \frac{\partial \Phi}{\partial \mathbf{F}}+J \mathbf{F}^{-1}\left\{\frac{1}{\mu_{o}}\left[\mathbb{B} \otimes \mathbb{B}-\frac{1}{2}(\mathbb{B} \cdot \mathbb{B}) \mathbf{I}\right]+\varepsilon_{o}\left[\mathbb{E} \otimes \mathbb{E}-\frac{1}{2}(\mathbb{E} \cdot \mathbb{E}) \mathbf{I}\right]\right\}$, 
where $\rho_{0}$ is the density at the reference configuration. From the above results it is possible to define the total energy function $\Omega$ (see $[13,28,24]$ ) as

$$
\Omega\left(\mathbf{F}, \mathbb{B}_{\mathrm{L}}, \mathbb{E}_{\mathrm{L}}\right)=\rho_{0} \Phi+\frac{1}{2 \mu_{o} J} \mathbb{B}_{\mathrm{L}} \cdot\left(\mathbf{C B}_{\mathrm{L}}\right)-\frac{\varepsilon_{o} J}{2} \mathbb{E}_{\mathrm{L}} \cdot\left(\mathbf{C}^{-1} \mathbb{E}_{\mathrm{L}}\right) .
$$

The Lagrangian polarization $\mathbb{P}_{\mathrm{L}}$ is defined as

$$
\mathbb{P}_{\mathrm{L}}=J \mathbf{F}^{-1} \mathbb{P},
$$

as a results from $(6)_{1},(15)_{3,4}$ we obtain

$$
\mathbb{D}_{\mathrm{L}}=\varepsilon_{o} \mathbf{C}^{-1} \mathbb{E}_{\mathrm{L}}+\mathbb{P}_{\mathrm{L}}
$$

Then using (16)-(18) in (14) 3 , we have

$$
\mathbb{D}_{\mathrm{L}}=-\frac{\partial \Omega}{\partial \mathbb{E}_{\mathrm{L}}}, \quad \mathbb{D}=-J^{-1} \mathbf{F} \frac{\partial \Omega}{\partial \mathbb{E}_{\mathrm{L}}}
$$

Let us define the Lagrangian magnetization $\mathbf{M}_{\mathrm{L}}$ as

$$
\mathbf{M}_{\mathrm{L}}=\mathbf{F}^{\mathrm{T}} \mathbf{M}
$$

and from $(6)_{2},(15)_{1,2}$ we obtain

$$
J^{-1} \mathrm{CB}_{\mathrm{L}}=\mu_{o}\left(\mathbb{H}_{\mathrm{L}}+\mathbb{M}_{\mathrm{L}}\right) .
$$

Furthermore, from (16), (20), (21) in (14) 3 we have

$$
\boldsymbol{H}_{\mathrm{L}}=\frac{\partial \Omega}{\partial \mathbb{B}_{\mathrm{L}}}, \quad \mathbb{H}=\mathbf{F}^{-\mathrm{T}} \frac{\partial \Omega}{\partial \mathbb{B}_{\mathrm{L}}}
$$

Finally, considering that $J^{-1} \mathbb{B}_{\mathrm{L}} \cdot\left(\mathrm{CB}_{\mathrm{L}}\right)=J \mathbb{B} \cdot \mathbb{B}$, and from the connections (see [27, 24]) $\frac{\partial}{\partial \mathbf{F}}(J \mathbb{B} \cdot \mathbb{B})=J \mathbf{F}^{-1}[2 \mathbb{B} \otimes \mathbb{B}-(\mathbb{B} \cdot \mathbb{B}) \mathbf{I}]$ and $\frac{\partial}{\partial \mathbf{F}}\left[J \mathbb{E}_{\mathrm{L}} \cdot\left(\mathbf{C}^{-1} \mathbb{E}_{\mathrm{L}}\right)\right]=-J \mathbf{F}^{-1}[2 \mathbb{E} \otimes \mathbb{E}-(\mathbb{E} \cdot \mathbb{E}) \mathbf{I}]$, from $(14)_{1},(16)$ we obtain

$$
\mathbf{T}=\frac{\partial \Omega}{\partial \mathbf{F}}, \quad \boldsymbol{\tau}=J^{-1} \mathbf{F} \frac{\partial \Omega}{\partial \mathbf{F}} .
$$

In the case of incompressible bodies $J=1$ from $(19)_{2},(23)$ we have

$$
\boldsymbol{\tau}=\mathbf{F} \frac{\partial \Omega}{\partial \mathbf{F}}-p \mathbf{I}, \quad \mathbb{D}=-\mathbf{F} \frac{\partial \Omega}{\partial \mathbb{E}_{\mathrm{L}}},
$$

where $p$ is the Lagrange multiplier connected with the constraint of incompressibility.

\section{Spectral constitutive equations}

In most of the applications concerning magneto-electro-elastic soft solids, the magneto-electroelastic effects can be increased considering transversely isotropic bodies, where there is a preferred direction (when the body is free of electromagnetic fields) commonly associated with particles aligned in that direction, where the electromagnetic effects are stronger, see Bastola and Hossain [3], Garcia-Gonzalez and Hossain [35]. If that direction is the unit vector field 
$\mathbf{a}_{0}$, then for the total energy function we have $\Omega\left(\mathbf{F}, \mathbb{B}_{\mathrm{L}}, \mathbb{E}_{\mathrm{L}}, \mathbf{a}_{0}\right)$. This function incorporates as a special case the situation when the body is isotropic. Since the above function must be frame-invariant and should be independent of the sign of the vectors fields we have

$$
\Omega\left(\mathbf{F}, \mathbb{B}_{\mathrm{L}}, \mathbb{E}_{\mathrm{L}}, \mathbf{a}_{0}\right)=W\left(\mathbf{C}, \boldsymbol{b} \otimes \mathfrak{b}, \mathbf{e} \otimes \mathbf{e}, \mathbf{a}_{0} \otimes \mathbf{a}_{0}, B_{\mathrm{L}}, E_{\mathrm{L}}\right),
$$

where

$$
B_{\mathrm{L}}=\sqrt{\mathbb{B}_{\mathrm{L}} \cdot \mathbb{B}_{\mathrm{L}}}, \quad E_{\mathrm{L}}=\sqrt{\mathbb{E}_{\mathrm{L}} \cdot \mathbb{E}_{\mathrm{L}}}, \quad \mathbb{B}_{\mathrm{L}}=B_{\mathrm{L}} \mathfrak{b}, \quad \mathbb{E}_{\mathrm{L}}=E_{\mathrm{L}} \mathbf{e},
$$

and $\mathbb{b}, \mathbb{e}$ are unit vector fields used for the definition of the magnetic and electric field variables, respectively.

If $\mathbf{U}$ is the right stretch tensor, we have the spectral representation $\mathbf{U}=\sum_{i=1}^{3} \lambda_{i} \mathbf{u}_{i} \otimes \mathbf{u}_{i}$, $\mathbf{C}=\mathbf{U}^{2}=\sum_{i=1}^{3} \lambda_{i}^{2} \mathbf{u}_{i} \otimes \mathbf{u}_{i}$, where $\lambda_{i}, \mathbf{u}_{i}, i=1,2,3$ are the principal stretches and principal directions, respectively. Then, from the spectral invariant theory developed by Shariff and co-workers $[19,20,66,67,69,71]$, we have

$$
W=W\left(\lambda_{1,2,3}, \aleph_{1,2,3}, \beth_{1,2,3}, \beth_{1,2,3}, B_{\mathrm{L}}, E_{\mathrm{L}}\right),
$$

where the notation $\lambda_{1,2,3}$ represents the set $\lambda_{1}, \lambda_{2}, \lambda_{3}$, and where we have defined

$$
\aleph_{i}=\mathbf{a}_{0} \cdot \mathbf{u}_{i}, \quad \beth_{i}=\mathfrak{b} \cdot \mathbf{u}_{i}, \quad \beth_{i}=\mathfrak{e} \cdot \mathbf{u}_{i},
$$

where

$$
\sum_{i=1}^{3} \aleph_{i}^{2}=1, \quad \sum_{i=1}^{3} \beth_{i}^{2}=1, \quad \sum_{i=1}^{3} \beth_{i}^{2}=1 .
$$

Note that the only 11 of the 14 invariants in (27) are independent. Since $W$ partially depends on the tensors $\mathbb{b} \otimes \mathfrak{b}, \mathbb{e} \otimes \mathbb{e}$ and $\mathbf{a}_{0} \otimes \mathbf{a}_{0}$, instead of the invariants in (27), the appropriate invariants for $W$ are

$$
\begin{aligned}
\lambda_{i}, \quad \alpha_{i}=\aleph_{i}^{2}, \quad \beta_{i}=\beth_{i}^{2}, \quad \gamma_{i}=\beth_{i}^{2}, \quad \zeta_{i}=\left(\mathbf{a}_{0} \cdot \mathfrak{b}\right) \aleph_{i} \mathfrak{b}_{i}, \\
\kappa_{i}=\left(\mathbf{a}_{0} \cdot \mathbf{e}\right) \aleph_{i} \mathbb{e}_{i}, \quad \varrho_{i}=(\mathbb{b} \cdot \mathbf{e}) \mathfrak{b}_{i} \mathbb{e}_{i}, \quad \eta_{i}=\left(\mathbf{a}_{0} \cdot \mathbb{b}\right)\left(\mathbf{a}_{0} \cdot \mathbf{e}\right) \mathfrak{b}_{i} \mathbb{e}_{i}, \\
\chi_{i}=\left(\mathbf{a}_{0} \cdot \mathbb{b}\right)(\mathbb{b} \cdot \mathbb{e}) \aleph_{i} \mathbb{e}_{i}, \quad \iota_{i}=(\mathbb{b} \cdot \mathbf{e})\left(\mathbf{a}_{0} \cdot \mathbb{e}\right) \aleph_{i} \mathfrak{b}_{i}, \quad B_{\mathrm{L}}, \quad E_{\mathrm{L}} .
\end{aligned}
$$

We strongly emphasize that $W$ must satisfy the P-property as described in [66, 67, 69, 71].

Using (27) in $(22)_{2},(24)$ we obtain (for an incompressible solid)

$$
\boldsymbol{\tau}=\mathbf{F} \frac{\partial W}{\partial \mathbf{C}} \mathbf{F}^{\mathrm{T}}-p \mathbf{I}, \quad \mathbb{H}=\mathbf{F}^{-\mathrm{T}} \frac{\partial W}{\partial \mathbb{B}_{\mathrm{L}}}, \quad \mathbb{D}=-\mathbf{F} \frac{\partial W}{\partial \mathbb{E}_{\mathrm{L}}} .
$$

The following expressions are needed in $(33)_{1}$

$$
\begin{aligned}
& \left(\frac{\partial W}{\partial \mathbf{C}}\right)_{i i}=\frac{1}{2 \lambda_{i}} \frac{\partial W}{\partial \lambda_{i}}, \\
& \left(\frac{\partial W}{\partial \mathbf{C}}\right)_{i j}=\frac{1}{2\left(\lambda_{i}^{2}-\lambda_{j}^{2}\right)}\left(\frac{\partial W}{\partial \mathbf{u}_{i}} \cdot \mathbf{u}_{j}-\frac{\partial W}{\partial \mathbf{u}_{j}} \cdot \mathbf{u}_{i}\right), \quad i \neq j .
\end{aligned}
$$

As well as this, for $(33)_{2,3}$ we need

$$
\begin{aligned}
& \left(\frac{\partial W}{\partial \mathbb{B}_{\mathrm{L}}}\right)_{i}=\frac{\partial W}{\partial B_{\mathrm{L}}} \beta_{i}+\frac{1}{B_{\mathrm{L}}}\left[(\mathbf{I}-\mathfrak{b} \otimes \mathfrak{b})^{\mathrm{T}} \frac{\partial W}{\partial \mathfrak{b}}\right] \cdot \mathbf{u}_{i}, \\
& \left(\frac{\partial W}{\partial \mathbb{E}_{\mathrm{L}}}\right)_{i}=\frac{\partial W}{\partial E_{\mathrm{L}}} \zeta_{i}+\frac{1}{E_{\mathrm{L}}}\left[(\mathbf{I}-\mathbf{e} \otimes \mathbf{e})^{\mathrm{T}} \frac{\partial W}{\partial \mathbf{e}}\right] \cdot \mathbf{u}_{i} .
\end{aligned}
$$


In terms of the invariants (30)-(32) the components $\left(\frac{\partial W}{\partial \mathbf{C}}\right)_{i j}, i \neq j$ become

$$
\begin{aligned}
\left(\frac{\partial W}{\partial \mathbf{C}}\right)_{i j}= & \frac{1}{\left(\lambda_{i}^{2}-\lambda_{j}^{2}\right)}\left[\left(\frac{\partial W}{\partial \alpha_{i}}-\frac{\partial W}{\partial \alpha_{j}}\right) \aleph_{i} \aleph_{j}+\left(\frac{\partial W}{\partial \beta_{i}}-\frac{\partial W}{\partial \beta_{j}}\right) \mathfrak{b}_{i} \mathfrak{b}_{j}\right. \\
& +\left(\frac{\partial W}{\partial \gamma_{i}}-\frac{\partial W}{\partial \gamma_{j}}\right) \mathbb{e}_{i} \mathbb{e}_{j}+\frac{1}{2}\left(\mathbf{a}_{0} \cdot \mathfrak{b}\right)\left(\frac{\partial W}{\partial \zeta_{i}}-\frac{\partial W}{\partial \zeta_{j}}\right)\left(\aleph_{i} \mathfrak{b}_{j}+\aleph_{j} \mathfrak{b}_{i}\right) \\
& +\frac{1}{2}\left(\mathbf{a}_{0} \cdot \mathbb{e}\right)\left(\frac{\partial W}{\partial \kappa_{i}}-\frac{\partial W}{\partial \kappa_{j}}\right)\left(\aleph_{i} \mathbb{e}_{j}+\aleph_{j} \mathbb{e}_{i}\right)+\frac{1}{2}(\mathfrak{b} \cdot \mathbf{e})\left(\frac{\partial W}{\partial \varrho_{i}}-\frac{\partial W}{\partial \varrho_{j}}\right)\left(\mathfrak{b}_{i} \mathbb{e}_{j}+\mathfrak{b}_{j} \mathbb{e}_{i}\right) \\
& +\frac{1}{2}\left(\mathbf{a}_{0} \cdot \mathfrak{b}\right)\left(\mathbf{a}_{0} \cdot \mathbf{e}\right)\left(\frac{\partial W}{\partial \eta_{i}}-\frac{\partial W}{\partial \eta_{j}}\right)\left(\mathfrak{b}_{i} \mathbb{e}_{j}+\mathbb{b}_{j} \mathbb{e}_{i}\right) \\
& +\frac{1}{2}\left(\mathbf{a}_{0} \cdot \mathfrak{b}\right)(\mathbb{b} \cdot \mathbf{e})\left(\frac{\partial W}{\partial \chi_{i}}-\frac{\partial W}{\partial \chi_{j}}\right)\left(\aleph_{i} \mathbb{e}_{j}+\aleph_{j} \mathbb{e}_{i}\right) \\
& \left.+\frac{1}{2}\left(\mathbf{a}_{0} \cdot \mathbf{e}\right)(\mathfrak{b} \cdot \mathbf{e})\left(\frac{\partial W}{\partial \iota_{i}}-\frac{\partial W}{\partial \iota_{j}}\right)\left(\aleph_{i} \mathfrak{b}_{j}+\aleph_{j} \mathfrak{b}_{i}\right)\right], \quad i \neq j .
\end{aligned}
$$

On the other hand

$$
\frac{\partial W}{\partial \mathfrak{b}}=\sum_{k=1}^{6} \mathbf{q}_{k}
$$

where we have defined

$$
\begin{aligned}
& \mathbf{q}_{1}=2 \sum_{i=1}^{3} \frac{\partial W}{\partial \beta_{i}} \mathfrak{b}_{i} \mathbf{u}_{i}, \quad \mathbf{q}_{2}=\sum_{i=1}^{3} \frac{\partial W}{\partial \zeta_{i}}\left[\aleph_{i} \mathfrak{b}_{i} \mathbf{a}_{0}+\left(\mathbf{a}_{0} \cdot \mathfrak{b}\right) \aleph_{i} \mathbf{u}_{i}\right], \\
& \mathbf{q}_{3}=\sum_{i=1}^{3} \frac{\partial W}{\partial \varrho_{i}}\left[\mathbb{b}_{i} \mathbb{e}_{i} \mathbf{e}+(\mathfrak{b} \cdot \mathbf{e}) \mathbb{e}_{i} \mathbf{u}_{i}\right], \quad \mathbf{q}_{4}=\sum_{i=1}^{3} \frac{\partial W}{\partial \eta_{i}}\left(\mathbf{a}_{0} \cdot \mathbf{e}\right)\left[\mathfrak{b}_{i} \mathbb{e}_{i} \mathbf{a}_{0}+\left(\mathbf{a}_{0} \cdot \mathfrak{b}\right) \mathbb{e}_{i} \mathbf{u}_{i}\right], \\
& \mathbf{q}_{5}=\sum_{i=1}^{3} \frac{\partial W}{\partial \chi_{i}}\left[(\mathbb{b} \cdot \mathbf{e}) \mathbf{a}_{0}+\left(\mathbf{a}_{0} \cdot \mathbb{b}\right) \mathbf{e}\right] \aleph_{i} \mathbb{e}_{i}, \quad \mathbf{q}_{6}=\sum_{i=1}^{3} \frac{\partial W}{\partial \iota_{i}}\left(\mathbf{a}_{0} \cdot \mathbf{e}\right)\left[\aleph_{i} \mathfrak{b}_{i} \mathbf{e}+(\mathbf{e} \cdot \mathfrak{b}) \aleph_{i} \mathbf{u}_{i}\right] .
\end{aligned}
$$

For the electric field, we have

$$
\frac{\partial W}{\partial \mathbf{e}}=\sum_{k=1}^{6} \mathbf{s}_{k},
$$

where

$$
\begin{aligned}
& \mathbf{s}_{1}=2 \sum_{i=1}^{3} \frac{\partial W}{\partial \gamma_{i}} \mathbb{e}_{i} \mathbf{u}_{i}, \quad \mathbf{s}_{2}=\sum_{i=1}^{3} \frac{\partial W}{\partial \kappa_{i}}\left[\aleph_{i} \mathbb{e}_{i} \mathbf{a}_{0}+\left(\mathbf{a}_{0} \cdot \mathbb{e}\right) \aleph_{i} \mathbf{u}_{i}\right], \\
& \mathbf{s}_{3}=\sum_{i=1}^{3} \frac{\partial W}{\partial \varrho_{i}}\left[\mathfrak{b}_{i} \mathbb{e}_{i} \mathfrak{b}+(\mathfrak{b} \cdot \mathbb{e}) \mathfrak{b}_{i} \mathbf{u}_{i}\right], \quad \mathbf{s}_{4}=\sum_{i=1}^{3} \frac{\partial W}{\partial \eta_{i}}\left(\mathbf{a}_{0} \cdot \mathfrak{b}\right)\left[\mathfrak{b}_{i} \mathbb{e}_{i} \mathbf{a}_{0}+\left(\mathbf{a}_{0} \cdot \mathbf{e}\right) \mathfrak{b}_{i} \mathbf{u}_{i}\right], \\
& \mathbf{s}_{5}=\sum_{i=1}^{3} \frac{\partial W}{\partial \iota_{i}}\left[(\mathbb{b} \cdot \mathbf{e}) \mathbf{a}_{0}+\left(\mathbf{e} \cdot \mathbf{a}_{0}\right) \mathfrak{b}\right] \aleph_{i} \mathfrak{b}_{i}, \quad \mathbf{s}_{6}=\sum_{i=1}^{3} \frac{\partial W}{\partial \chi_{i}}\left(\mathbf{a}_{0} \cdot \mathfrak{b}\right)\left[\aleph_{i} \mathbb{e}_{i} \mathfrak{b}+(\mathbb{b} \cdot \mathbb{e}) \aleph_{i} \mathbf{u}_{i}\right] .
\end{aligned}
$$

\section{Boundary value problems}

We study five boundary value problems, four of them correspond to problems wherein the strains, stresses and electromagnetic variables are homogeneous inside the body, and one 
problem with non-homogeneous distributions. In all these cases we only study incompressible bodies. In the case of bodies with homogeneous deformations and electromagnetic field, the Maxwell equations (2) and the equation of equilibrium (when there is no mechanical body load) (10) are satisfied automatically.

\subsection{Uniform extension/compression of a cylinder}

Consider a cylinder defined in the reference configuration in cylindrical coordinates as

$$
0 \leq R \leq R_{\mathrm{o}}, \quad 0 \leq \Theta \leq 2 \pi, \quad 0 \leq Z \leq L,
$$

where it is assumed that

$$
\mathbf{a}_{0}=\mathbf{E}_{Z}, \quad \mathbb{E}=E_{o} \mathbf{e}_{z}, \quad \mathbb{B}=B_{o} \mathbf{e}_{z},
$$

and $E_{o}$ and $B_{o}$ are constants. The deformation is

$$
r=\lambda_{r} R, \quad \theta=\Theta, \quad z=\lambda_{z} Z,
$$

where $\lambda_{r}>0$ and $\lambda_{z}>0$ are constants. The deformation gradient is

$$
\mathbf{F}=\lambda_{r} \mathbf{e}_{r} \otimes \mathbf{E}_{R}+\lambda_{r} \mathbf{e}_{\theta} \otimes \mathbf{E}_{\Theta}+\lambda_{z} \mathbf{e}_{z} \otimes \mathbf{E}_{Z}
$$

Therefore

$$
\lambda_{1}=\lambda_{2}=\lambda_{r}, \quad \lambda_{3}=\lambda_{z}, \quad \mathbf{u}_{1}=\mathbf{E}_{R}, \quad \mathbf{u}_{2}=\mathbf{E}_{\Theta}, \quad \mathbf{u}_{3}=\mathbf{E}_{Z},
$$

where $\operatorname{det} \mathbf{F}=1$ implies that $\lambda_{r}=\frac{1}{\sqrt{\lambda_{z}}}$. Using (48) and (51) $)_{3,4,5}$ in (28), (30)-(32), it is obtained

$$
\begin{aligned}
& \aleph_{1}=\aleph_{2}=0, \quad \aleph_{3}=1, \quad \beth_{1}=\beth_{2}=0, \quad \beth_{3}=1, \quad \beth_{1}=I_{2}=0, \quad I_{3}=1, \\
& \alpha_{1}=\alpha_{2}=0, \quad \alpha_{3}=1, \quad \beta_{1}=\beta_{2}=0, \quad \beta_{3}=1, \quad \gamma_{1}=\gamma_{2}=0, \quad \gamma_{3}=1 \text {, } \\
& \zeta_{1}=\zeta_{2}=0, \quad \zeta_{3}=1, \quad \kappa_{1}=\kappa_{2}=0, \quad \kappa_{3}=1, \quad \varrho_{1}=\varrho_{2}=0, \quad \varrho_{3}=1, \\
& \eta_{1}=\eta_{2}=0, \quad \eta_{3}=1, \quad \chi_{1}=\chi_{2}=0, \quad \chi_{3}=1, \quad \iota_{1}=\iota_{2}=0, \quad \iota_{3}=1 .
\end{aligned}
$$

Using (48) and (50) from (15), (26), we have

$$
\mathbb{B}_{\mathrm{L}}=\lambda_{3}^{-1} B_{o} \mathbf{E}_{Z}, \quad B_{\mathrm{L}}=\lambda_{3}^{-1} B_{o}, \quad \mathbb{b}=\mathbf{E}_{Z}, \quad \mathbb{E}_{\mathrm{L}}=\lambda_{3} E_{o} \mathbf{E}_{Z}, \quad E_{\mathrm{L}}=\lambda_{3} E_{o}, \quad \mathbb{e}=\mathbf{E}_{Z} .
$$

From (3), (34), (35) and (38), we obtain

$$
\tau_{r r}=\tau_{\theta \theta}=\lambda_{1} \frac{\partial W}{\partial \lambda_{1}}-p, \quad \tau_{z z}=\lambda_{3} \frac{\partial W}{\partial \lambda_{3}}-p,
$$

where $\tau_{i j}=0$ if $i \neq j$. On the other hand from (39) and (43), we have

$$
\frac{\partial W}{\partial \mathbf{b}}=2\left(\frac{\partial W}{\partial \beta_{3}}+\frac{\partial W}{\partial \zeta_{3}}+\frac{\partial W}{\partial \varrho_{3}}+\frac{\partial W}{\partial \eta_{3}}+\frac{\partial W}{\partial \chi_{3}}+\frac{\partial W}{\partial \iota_{3}}\right) \mathbf{E}_{Z}
$$

and

$$
\frac{\partial W}{\partial \mathbf{e}}=2\left(\frac{\partial W}{\partial \gamma_{3}}+\frac{\partial W}{\partial \kappa_{3}}+\frac{\partial W}{\partial \varrho_{3}}+\frac{\partial W}{\partial \eta_{3}}+\frac{\partial W}{\partial \chi_{3}}+\frac{\partial W}{\partial \iota_{3}}\right) \mathbf{E}_{Z}
$$


and from (36), (37) this implies that $\left(\frac{\partial W}{\partial \mathbb{B}_{\mathrm{L}}}\right)_{1}=\left(\frac{\partial W}{\partial \mathbb{B}_{\mathrm{L}}}\right)_{2}=0,\left(\frac{\partial W}{\partial \mathbb{B}_{\mathrm{L}}}\right)_{3}=\frac{\partial W}{\partial B_{\mathrm{L}}}$ and $\left(\frac{\partial W}{\partial \mathbb{E}_{\mathrm{L}}}\right)_{1}=$ $\left(\frac{\partial W}{\partial \mathbb{E}_{\mathrm{L}}}\right)_{2}=0,\left(\frac{\partial W}{\partial \mathbb{E}_{\mathrm{L}}}\right)_{3}=\frac{\partial W}{\partial E_{\mathrm{L}}}$. Furthermore, as results from $(33)_{2,3}$, it is obtained

$$
\mathbb{H}=\mathbb{H}_{z} \mathbf{e}_{z}=\lambda_{3}^{-1} \frac{\partial W}{\partial B_{\mathrm{L}}} \mathbf{e}_{z}, \quad \mathbb{D}=\mathbb{D}_{z} \mathbf{e}_{z}=-\lambda_{3} \frac{\partial W}{\partial E_{\mathrm{L}}} \mathbf{e}_{z} .
$$

Regarding the continuity conditions, assuming that $L \gg R_{\mathrm{o}}$, the conditions are only studied for the surface $R=R_{\mathrm{o}}$, where $\mathbf{n}=\mathbf{e}_{r}$. Considering $(48)_{2,3}$, let us assume that the electric field and magnetic induction outside in vacuum are

$$
\mathbb{E}^{(\mathrm{o})}=E_{o}^{(\mathrm{o})} \mathbf{e}_{z}, \quad \mathbb{B}^{(\mathrm{o})}=B_{o}^{(\mathrm{o})} \mathbf{e}_{z},
$$

where $E_{o}^{(\mathrm{o})}$ and $B_{o}^{(\mathrm{o})}$ are constants. From $(48)_{3},(59)_{2}$ the continuity condition $(11)_{1}$ is satisfied automatically, whereas from $(48)_{2},(59)_{1}$ the continuity condition $(11)_{2}$ is satisfied if

$$
E_{o}^{(\mathrm{o})}=E_{o} .
$$

On the other hand from $(59)_{1}$ and $(5)_{2}$ we have

$$
\mathrm{H}^{(\mathrm{o})}=\mu_{o} B_{o}^{(\mathrm{o})} \mathbf{e}_{z} .
$$

From $(58)_{1},(61)$ the continuity condition $(11)_{4}$ is satisfied if $\uplus_{z}=\frac{1}{\mu_{o}} B_{o}^{(o)}$ which from (58) means that

$$
\lambda_{z}^{-1} \frac{\partial W}{\partial B_{\mathrm{L}}}=\frac{1}{\mu_{o}} B_{o}^{(o)} .
$$

The above equation can be used, for example, to obtain $B_{o}$ for a given $B_{o}^{(\mathrm{o})}$.

Regarding the electric field inside the cylinder, from the practical point of view it can be generated with electrodes attached on the surfaces $Z=0$ and $Z=L$ by placing an electric potential $\Phi=\Phi(Z)$ such that $E_{o}=-\frac{[\Phi(L)-\Phi(0)]}{L}=-\frac{\Delta \Phi}{L}$.

Finally, from (59) and (13) the radial component of the Maxwell stress is $\tau_{\mathrm{M}_{r r}}=-\frac{\varepsilon_{o} E_{o}^{2}}{2}-$ $\frac{B_{o}^{(o)^{2}}}{2 \mu_{o}}$. As a result, if on the lateral surface $R=R_{\mathrm{o}}$ there is no mechanical load, from (12) we obtain $\tau_{r r}=\tau_{\mathrm{M}_{r r}}$ and from (57) 1 we have

$$
p=\lambda_{1} \frac{\partial W}{\partial \lambda_{1}}+\frac{\varepsilon_{o} E_{o}^{2}}{2}+\frac{\left.B_{o}^{(o)}\right)^{2}}{2 \mu_{o}} .
$$

Regarding the surfaces $Z=0$ and $Z=L$, it is assumed that an external 'total' load $\hat{t}_{z} \mathbf{e}_{z}$ is applied, which incorporates the effect of the Maxwell stresses, therefore, from (12) we obtain $\tau_{z z}=\hat{t}_{z}$ and using (63) in $(57)_{2}$, finally it is obtained

$$
\hat{t}_{z}=\lambda_{3} \frac{\partial W}{\partial \lambda_{3}}-\lambda_{1} \frac{\partial W}{\partial \lambda_{1}}-\frac{\varepsilon_{o} E_{o}^{2}}{2}-\frac{B_{o}^{(o)^{2}}}{2 \mu_{o}} .
$$

\subsection{Uniform extension of a thin slab}

Consider the prismatic bar

$$
-\frac{L_{i}}{2} \leq X_{i} \leq \frac{L_{i}}{2}, \quad i=1,2,3
$$


where it is assumed that $L_{3} \ll L_{1}$ and $L_{3} \ll L_{2}$. In this problem the external mechanical load is applied on the surfaces $X_{3}= \pm \frac{L_{3}}{2}$. Regarding the electric field and magnetic induction it is assumed that

$$
\mathbb{B}=B_{o} \mathbf{e}_{3}, \quad \mathbb{E}=E_{o} \mathbf{e}_{3},
$$

where $B_{o}$ and $E_{o}$ are constants. For the vector field $\mathbf{a}_{0}$ two cases are studied:

$$
\mathbf{a}_{0}=\mathbf{E}_{3}, \quad \mathbf{a}_{0}=\mathbf{E}_{1} .
$$

The motion is assumed to be

$$
x_{i}=\lambda_{i} X_{i}, \quad i=1,2,3,
$$

where $\lambda_{i}>0$ and $\lambda_{1} \lambda_{2} \lambda_{3}=1$, then

$$
\mathbf{F}=\sum_{i=1}^{3} \lambda_{i} \mathbf{e}_{i} \otimes \mathbf{E}_{i}, \quad \mathbf{u}_{i}=\mathbf{E}_{i} .
$$

From (15), (66) and (69), (26) we have

$$
B_{\mathrm{L}}=\lambda_{3}^{-1} B_{o}, \quad E_{\mathrm{L}}=\lambda_{3} E_{o}, \quad \boldsymbol{b}=\mathbf{E}_{3}, \quad \mathbb{e}=\mathbf{E}_{3} .
$$

From (33)-(35), it is easy to show that

$$
\tau_{i i}=\lambda_{i} \frac{\partial W}{\partial \lambda_{i}}-p, \quad \tau_{i j}=0 \quad i \neq j
$$

\subsubsection{Case $\mathrm{a}_{0}=\mathbf{E}_{3}$}

Using (67) 1 and (70), (69) 2 in (28), (30)-(32) the same invariants as in (52)-(55) are obtained. On the other hand, from $(33)_{2,3}$ considering (36), (37), (39), (43) and (66), we have

$$
\mathbb{H}=\mathbb{H}_{3} \mathbf{e}_{3}=\lambda_{3}^{-1} \frac{\partial W}{\partial B_{\mathrm{L}}} \mathbf{e}_{3}, \quad \mathbb{D}=\mathbb{D}_{3} \mathbf{e}_{3}=-\lambda_{3} \frac{\partial W}{\partial E_{\mathrm{L}}} \mathbf{e}_{3} .
$$

Let us assume that the electric field and magnetic induction outside the slab are given as

$$
\mathbb{E}^{(\mathrm{o})}=E_{o}^{(\mathrm{o})} \mathbf{e}_{3}, \quad \mathbb{B}^{(\mathrm{o})}=B_{o}^{(\mathrm{o})} \mathbf{e}_{3},
$$

where $E_{o}^{(\mathrm{o})}$ and $B_{o}^{(\mathrm{o})}$ are constants. Accordingly, for the surfaces $X_{3}= \pm \frac{L_{3}}{2}$, the continuity condition $(11)_{2}$ is satisfied automatically, while $(11)_{1}$ from $(73)_{2},(66)_{1}$ is satisfied if

$$
B_{o}^{(\mathrm{o})}=B_{o} .
$$

From (73) and (5) we obtain

$$
\mathbb{H}^{(\mathrm{o})}=\frac{1}{\mu_{o}} B_{o}^{(\mathrm{o})} \mathbf{e}_{3}, \quad \mathbb{D}^{(\mathrm{o})}=\varepsilon_{o} E_{o}^{(\mathrm{o})} \mathbf{e}_{3},
$$

and the continuity condition $(11)_{3}$ is satisfied if

$$
-\lambda_{3} \frac{\partial W}{\partial E_{\mathrm{L}}}=\varepsilon_{o} E_{o}^{(\mathrm{o})},
$$


whereas $(11)_{4}$ is satisfied automatically.

In the expressions for the components of the total stress (71) we have $p$, which should be obtained from the boundary conditions. Let us assume that for the surface $X_{1}= \pm \frac{L_{1}}{2}$, there is no mechanical load, then considering (73) from (13), we have $\tau_{\mathrm{M}_{11}}=-\frac{\varepsilon_{o} E_{o}^{2}}{2}-\frac{B_{o}^{2}}{2 \mu_{o}}$. Furthermore, the continuity condition (12) becomes $\tau_{11}=\tau_{\mathrm{M}_{11}}$ and from (71) using the above condition, we obtain the Lagrange multiplier as

$$
p=\lambda_{1} \frac{\partial W}{\partial \lambda_{1}}+\frac{\varepsilon_{o} E_{o}^{2}}{2}+\frac{B_{o}{ }^{2}}{2 \mu_{o}} .
$$

On the surfaces $X_{3}= \pm \frac{L_{3}}{2}$, if the external load is $\hat{t}_{3} \mathbf{e}_{3}$ (which we assume it incorporates the Maxwell stresses), from (12) we have $\tau_{33}=\hat{t}_{3}$, from where the following expression is finally obtained (see (71))

$$
\hat{t}_{3}=\lambda_{3} \frac{\partial W}{\partial \lambda_{3}}-\lambda_{1} \frac{\partial W}{\partial \lambda_{1}}-\frac{\varepsilon_{o} E_{o}^{2}}{2}-\frac{B_{o}^{2}}{2 \mu_{o}} .
$$

\subsubsection{Case $\mathrm{a}_{0}=\mathrm{E}_{1}$}

In this case, several of the equations presented in Section 4.2.1 are the same, in particular (72) and (78). The difference corresponds to the values of some of the invariants, which are:

$$
\begin{aligned}
& \aleph_{1}=1, \quad \aleph_{2}=\aleph_{3}=0, \quad \alpha_{1}=1, \quad \alpha_{2}=\alpha_{3}=0, \quad \zeta_{i}=0, \quad \kappa_{i}=0, \\
& \eta_{i}=0, \quad \chi_{i}=0, \quad \iota_{i}=0 .
\end{aligned}
$$

\subsection{Biaxial deformation of a thin plate}

For the same body described in (65) now under the assumption that $L_{1} \ll L_{2}, L_{1} \ll L_{3}$ (which means it is a thin plate), we consider the following cases

$$
\mathbf{a}_{0}=\left\{\begin{array}{l}
\mathbf{E}_{1}, \\
\mathbf{E}_{2}, \\
\mathbf{E}_{3},
\end{array}, \quad \mathbb{E}=\left\{\begin{array}{l}
E_{o} \mathbf{e}_{1}, \\
E_{o} \mathbf{e}_{3},
\end{array}, \quad \mathbb{B}=\left\{\begin{array}{l}
B_{o} \mathbf{e}_{1}, \\
B_{o} \mathbf{e}_{2}, \\
B_{o} \mathbf{e}_{3},
\end{array}\right.\right.\right.
$$

where $E_{o}$ and $B_{o}$ are constants. In this problem, the same motion is assumed as in (68) $x_{i}=\lambda_{i} X_{i}$, where $\lambda_{1} \lambda_{2} \lambda_{3}=1$, as a result $\mathbf{u}_{i}=\mathbf{E}_{i}$.

\subsubsection{Case $\mathbf{a}_{0}=\mathbf{E}_{3}, \mathbb{E}=E_{o} \mathbf{e}_{3}$ and $\mathbb{B}=B_{o} \mathbf{e}_{3}$}

This problem is very similar to the case presented in Section 4.2.1, in particular the relations (71) and (72) are the same. The difference corresponds to the continuity conditions. Because of the assumption $L_{1} \ll L_{2}$ and $L_{1} \ll L_{3}$, the continuity conditions are only analyzed for the surfaces $X_{1}= \pm \frac{L_{1}}{2}$. Considering that $\mathbb{E}=E_{o} \mathbf{e}_{3}$ and $\mathbb{B}=B_{o} \mathbf{e}_{3}$ we assume that (see (73))

$$
\mathbb{E}^{(\mathrm{o})}=E_{o}^{(\mathrm{o})} \mathbf{e}_{3}, \quad \mathbb{B}^{(\mathrm{o})}=B_{o}^{(\mathrm{o})} \mathbf{e}_{3} .
$$

Then the continuity condition $(11)_{1}$ is satisfied automatically, while $(11)_{2}$ is satisfied if

$$
E_{o}^{(\mathrm{o})}=E_{o} .
$$


From (5) and (82), we have

$$
\mathbb{D}^{(\mathrm{o})}=\varepsilon_{o} E_{o} \mathbf{e}_{3}, \quad \mathbb{H}^{(\mathrm{o})}=\frac{1}{\mu_{o}} B_{o}^{(\mathrm{o})} \mathbf{e}_{3},
$$

and the continuity condition $(11)_{3}$ is satisfied automatically, whereas $(11)_{4}$ implies that

$$
\lambda_{3}^{-1} \frac{\partial W}{\partial B_{\mathrm{L}}}=\frac{1}{\mu_{o}} B_{o}^{(o)}
$$

From this, it can be obtained, for example, $B_{o}$ in terms of $B_{o}^{(o)}$. Using (82) in (13), we have $\tau_{\mathrm{M}_{11}}=-\frac{\varepsilon_{o} E_{o}^{2}}{2}-\frac{B_{o}^{(o)^{2}}}{2 \mu_{o}}$. Assuming that on the surfaces $X_{1}= \pm \frac{L_{1}}{2}$ there is no external mechanical load which, from (12), results in $\tau_{11}=\tau_{\mathrm{M}_{11}}$. Furthermore, considering (71) we obtain

$$
p=\lambda_{1} \frac{\partial W}{\partial \lambda_{1}}+\frac{\varepsilon_{o} E_{o}^{2}}{2}+\frac{B_{o}^{(\mathrm{o})^{2}}}{2 \mu_{o}} .
$$

Let us assume that on the surfaces $X_{2}= \pm \frac{L_{2}}{2}$ and $X_{3}= \pm \frac{L_{3}}{2}$, we apply external loads (that as before incorporate the Maxwell stresses) $\hat{t}_{2} \mathbf{e}_{2}$ and $\hat{t}_{3} \mathbf{e}_{3}$, respectively. Then from (12) we have $\tau_{22}=\hat{t}_{2}$ and $\tau_{33}=\hat{t}_{3}$ and considering (71) and (86), we finally obtain

$$
\hat{t}_{2}=\lambda_{2} \frac{\partial W}{\partial \lambda_{2}}-\lambda_{1} \frac{\partial W}{\partial \lambda_{1}}-\frac{\varepsilon_{o} E_{o}^{2}}{2}-\frac{B_{o}^{(o)^{2}}}{2 \mu_{o}}, \quad \hat{t}_{3}=\lambda_{3} \frac{\partial W}{\partial \lambda_{3}}-\lambda_{1} \frac{\partial W}{\partial \lambda_{1}}-\frac{\varepsilon_{o} E_{o}^{2}}{2}-\frac{B_{o}^{(o)^{2}}}{2 \mu_{o}} .
$$

\subsubsection{Case $\mathbf{a}_{0}=\mathbf{E}_{3}, \mathbb{E}=E_{o} \mathbf{e}_{3}$ and $\mathbb{B}=B_{o} \mathbf{e}_{1}$}

In this problem $(70)_{1,2,5,6},(53)_{1,2,4,6},(54),(55)$ are the same, while

$$
\beth_{1}=1, \quad \beth_{2}=\beth_{3}=0, \quad \beta_{1}=1, \quad \beta_{2}=\beta_{3}=0 .
$$

Similarly from (15) and (26), we have

$$
\mathbb{B}_{\mathrm{L}}=\lambda_{1}^{-1} B_{o} \mathbf{E}_{Z}, \quad B_{\mathrm{L}}=\lambda_{1}^{-1} B_{o}, \quad \mathfrak{b}=\mathbf{E}_{1}, \quad \mathbb{E}_{\mathrm{L}}=\lambda_{3} E_{o} \mathbf{E}_{3}, \quad E_{\mathrm{L}}=\lambda_{3} E_{o}, \quad \mathbb{e}=\mathbf{E}_{3} .
$$

On the other hand, from (39) and (43) we obtain $\frac{\partial W}{\partial \mathbf{b}}=2 \frac{\partial W}{\partial \beta_{1}} \mathbf{E}_{1}$ and $\frac{\partial W}{\partial \mathbf{e}}=2\left(\frac{\partial W}{\partial \gamma_{3}}+\frac{\partial W}{\partial \kappa_{3}}\right) \mathbf{E}_{3}$. This implies that $\left(\frac{\partial W}{\partial \mathbb{B}_{\mathrm{L}}}\right)_{1}=\frac{\partial W}{\partial B_{\mathrm{L}}},\left(\frac{\partial W}{\partial \mathbb{B}_{\mathrm{L}}}\right)_{2}=\left(\frac{\partial W}{\partial \mathbb{B}_{\mathrm{L}}}\right)_{3}=0$ and $\left(\frac{\partial W}{\partial \mathbb{E}_{\mathrm{L}}}\right)_{1}=\left(\frac{\partial W}{\partial \mathbb{E}_{\mathrm{L}}}\right)_{2}=0$, $\left(\frac{\partial W}{\partial \mathbb{E}_{\mathrm{L}}}\right)_{3}=\frac{\partial W}{\partial E_{\mathrm{L}}}$. Furthermore, from (36), (37), (33) 2,3 we have

$$
\mathbb{H}=\lambda_{1}^{-1} \frac{\partial W}{\partial B_{\mathrm{L}}} \mathbf{e}_{1}, \quad \mathbb{D}=-\lambda_{3} \frac{\partial W}{\partial E_{\mathrm{L}}} \mathbf{e}_{3} .
$$

The continuity conditions (11) are analyzed for the surfaces $X_{1}= \pm \frac{L_{1}}{2}$. Let us assume that

$$
\mathbb{B}^{(o)}=B_{o}^{(o)} \mathbf{e}_{1}, \quad \mathbb{E}^{(o)}=E_{o}^{(o)} \mathbf{e}_{3},
$$

then $(11)_{2}$ is satisfied if $E_{o}^{(\mathrm{o})}=E_{o}$, and $(11)_{1}$ holds if $B_{o}^{(\mathrm{o})}=B_{o}$. From (5) we obtain

$$
\mathbb{H}^{(\mathrm{o})}=\frac{1}{\mu_{o}} B_{o} \mathbf{e}_{1}, \quad \mathbb{D}^{(\mathrm{o})}=\varepsilon_{o} E_{o} \mathbf{e}_{3},
$$


and $(11)_{3,4}$ are satisfied automatically.

Finally, using (91) in (13) we obtain $\tau_{\mathrm{M}_{11}}=-\frac{\varepsilon_{o}}{2} E_{o}^{2}+\frac{1}{2 \mu_{o}} B_{o}^{2}$, which can be replaced in (12) assuming that on the surfaces $X_{1}= \pm \frac{L_{1}}{2}$ there is no mechanical traction. Moreover, using (71) (which are the same for this problem) we can obtain $p$. In a similar manner as the problem studied in the previous section, we obtain for the traction applied on the surfaces $X_{2}= \pm \frac{L_{2}}{2}, X_{3}= \pm \frac{L_{3}}{2}$, respectively:

$$
\hat{t}_{2}=\lambda_{2} \frac{\partial W}{\partial \lambda_{2}}-\lambda_{1} \frac{\partial W}{\partial \lambda_{1}}-\frac{\varepsilon_{o}}{2} E_{o}^{2}+\frac{1}{2 \mu_{o}} B_{o}^{2}, \quad \hat{t}_{3}=\lambda_{3} \frac{\partial W}{\partial \lambda_{3}}-\lambda_{1} \frac{\partial W}{\partial \lambda_{1}}-\frac{\varepsilon_{o}}{2} E_{o}^{2}+\frac{1}{2 \mu_{o}} B_{o}^{2} .
$$

\subsubsection{Case $\mathbf{a}_{0}=\mathbf{E}_{3}, \mathbb{E}=E_{o} \mathbf{e}_{1}$ and $\mathbb{B}=B_{o} \mathbf{e}_{1}$}

For this case from (28), (30)-(32), we have

$$
\begin{aligned}
& \aleph_{1}=\aleph_{2}=0, \quad \aleph_{3}=1, \quad \beth_{1}=1, \quad \beth_{2}=\beth_{3}=0, \quad \beth_{1}=1, \quad \beth_{2}=\beth_{3}=0, \\
& \alpha_{1}=\alpha_{2}=0, \quad \alpha_{3}=1, \quad \beta_{1}=1, \quad \beta_{2}=\beta_{3}=0, \quad \gamma_{1}=1, \quad \gamma_{2}=\gamma_{3}=0, \\
& \zeta_{i}=0, \quad \kappa_{i}=0, \quad \varrho_{1}=1, \quad \varrho_{2}=\varrho_{3}=0, \quad \eta_{i}=0, \quad \chi_{i}=0, \quad \iota_{i}=0 .
\end{aligned}
$$

From $(33)_{1},(34),(35)$ the same expressions (71) for the components of the total stress are valid. On the other hand, from (39), (43) we obtain $\frac{\partial W}{\partial \mathbf{b}}=2\left(\frac{\partial W}{\partial \beta_{1}}+\frac{\partial W}{\partial \varrho_{1}}\right) \mathbf{E}_{1}$ and $\frac{\partial W}{\partial \mathbf{e}}=$ $2\left(\frac{\partial W}{\partial \gamma_{1}}+\frac{\partial W}{\partial \varrho_{1}}\right) \mathbf{E}_{1}$, and from $(33)_{2,3},(36),(37)$ we have

$$
\boldsymbol{H}=\lambda_{1}^{-1} \frac{\partial W}{\partial B_{\mathrm{L}}} \mathbf{e}_{1}, \quad \mathbb{D}=-\lambda_{1} \frac{\partial W}{\partial E_{\mathrm{L}}} \mathbf{e}_{1} .
$$

Let us assume that the magnetic induction and electric field outside the plate are given as

$$
\mathbb{B}^{(o)}=B_{o}^{(o)} \mathbf{e}_{1}, \quad \mathbb{E}^{(o)}=E_{o}^{(o)} \mathbf{e}_{1},
$$

where $B_{o}^{(o)}$, and $E_{o}^{(o)}$ are constants, then $(11)_{1}$ is satisfied if

$$
B_{o}^{(o)}=B_{o}
$$

while $(11)_{2}$ is satisfied automatically. From (5) and (98) we have $\mathbb{D}^{(\mathrm{o})}=\varepsilon_{o} E_{o}^{(\mathrm{o})} \mathbf{e}_{1}$ and $\mathbb{H}^{(\mathrm{o})}=$ $\frac{1}{\mu_{o}} B_{o} \mathbf{e}_{1}$. Then, it is easy to see that $(11)_{4}$ is satisfied automatically, whereas $(11)_{3}$ holds if

$$
-\lambda_{1} \frac{\partial W}{\partial E_{\mathrm{L}}}=\varepsilon_{o} E_{o}^{(\mathrm{o})}
$$

from where we can obtain, for example, $E_{o}$ in terms of $E_{o}^{(\mathrm{o})}$. Finally, from (98) and (13) we have $\tau_{\mathrm{M}_{11}}=-\frac{\varepsilon_{o}}{2} E_{o}^{(\mathrm{o})^{2}}-\frac{B_{o}^{2}}{2 \mu_{o}}$ and using this in (12) following the same procedure as in the previous Section 4.2.1 we obtain the external total loads on the surfaces $X_{2}= \pm \frac{L_{2}}{2}$ and $X_{3}= \pm \frac{L_{3}}{2}$ that are necessary to produce the deformation

$$
\hat{t}_{2}=\lambda_{2} \frac{\partial W}{\partial \lambda_{2}}-\lambda_{1} \frac{\partial W}{\partial \lambda_{1}}+\frac{\varepsilon_{o}}{2} E_{o}^{(\mathrm{o})^{2}}+\frac{B_{o}^{2}}{2 \mu_{o}}, \quad \hat{t}_{3}=\lambda_{3} \frac{\partial W}{\partial \lambda_{3}}-\lambda_{1} \frac{\partial W}{\partial \lambda_{1}}+\frac{\varepsilon_{o}}{2} E_{o}^{(\mathrm{o})^{2}}+\frac{B_{o}^{2}}{2 \mu_{o}} .
$$




\subsubsection{Case $\mathbf{a}_{0}=\mathbf{E}_{3}, \mathbb{E}=E_{o} \mathbf{e}_{1}$ and $\mathbb{B}=B_{o} \mathbf{e}_{3}$}

In this problem from (28), (30)-(32), we obtain

$$
\begin{aligned}
& \aleph_{1}=\aleph_{2}=0, \quad \aleph_{3}=1, \quad \beth_{1}=\beth_{2}=0, \quad \beth_{3}=1, \quad \beth_{1}=1, \quad \beth_{2}=\beth_{3}=0, \\
& \alpha_{1}=\alpha_{2}=0, \quad \alpha_{3}=1, \quad \beta_{1}=\beta_{2}=0, \quad \beta_{3}=1, \quad \gamma_{1}=1, \quad \gamma_{2}=\gamma_{3}=0, \\
& \zeta_{1}=1, \quad \zeta_{2}=\zeta_{3}=0, \quad \kappa_{i}=0, \quad \varrho_{i}=0, \quad \eta_{i}=0, \quad \chi_{i}=0, \quad \iota_{i}=0 .
\end{aligned}
$$

As in the previous problems the same expressions for the components of the total stress (71) are valid here. As well as this from (39), (43) we have $\frac{\partial W}{\partial \mathbf{b}}=2\left(\frac{\partial W}{\partial \beta_{3}}+\frac{\partial W}{\partial \zeta_{3}}\right) \mathbf{E}_{3}$ and $\frac{\partial W}{\partial \mathbf{e}}=2 \frac{\partial W}{\partial \gamma_{1}} \mathbf{E}_{1}$. Therefore, from $(33)_{2,3}$ we obtain

$$
\mathbb{H}=\lambda_{3}^{-1} \frac{\partial W}{\partial B_{\mathrm{L}}} \mathbf{e}_{3}, \quad \mathbb{D}=-\lambda_{1} \frac{\partial W}{\partial E_{\mathrm{L}}} \mathbf{e}_{1} .
$$

Let us assume that the magnetic induction and electric field in vacuum are

$$
\mathbb{B}^{(o)}=B_{o}^{(o)} \mathbf{e}_{3}, \quad \mathbb{E}^{(o)}=E_{o}^{(o)} \mathbf{e}_{1},
$$

then $(11)_{1,2}$ are satisfied automatically, and from (5) we obtain $\mathbf{H}^{(o)}=\frac{1}{\mu_{o}} B_{o}^{(o)} \mathbf{e}_{3}$ and $\mathbb{D}^{(o)}=$ $\varepsilon_{o} E_{o}^{(o)} \mathbf{e}_{1}$, and from (105) the continuity conditions (11) $)_{3,4}$ are satisfied if

$$
\lambda_{3}^{-1} \frac{\partial W}{\partial B_{\mathrm{L}}}=\frac{1}{\mu_{o}} B_{o}^{(\mathrm{o})}, \quad-\lambda_{1} \frac{\partial W}{\partial E_{\mathrm{L}}}=\varepsilon_{o} E_{o}^{(\mathrm{o})}
$$

from where we can obtain, for example, $B_{o}$ and $E_{o}$ in terms of $B_{o}^{(\mathrm{o})}$ and $E_{o}^{(\mathrm{o})}$. Using (106) in (13) we have $\tau_{\mathrm{M}_{11}}=\frac{\varepsilon_{o}}{2} E_{o}^{(\mathrm{o})^{2}}-\frac{1}{2 \mu_{o}} B_{o}^{(\mathrm{o})^{2}}$. Assuming that on the surfaces $X_{1}= \pm \frac{L_{1}}{2}$, there is no external mechanical traction, for which, from (12), (71) we obtain $p=\lambda_{1} \frac{\partial W}{\partial \lambda_{1}}-\frac{\varepsilon_{o}}{2} E_{o}^{(o)^{2}}+$ $\frac{1}{2 \mu_{o}} B_{o}^{(\mathrm{o})^{2}}$. Therefore, the external total traction that is necessary to apply on the surfaces $X_{2}= \pm \frac{L_{2}}{2}$ and $X_{3}= \pm \frac{L_{3}}{2}$ are

$$
\hat{t}_{2}=\lambda_{2} \frac{\partial W}{\partial \lambda_{2}}-\lambda_{1} \frac{\partial W}{\partial \lambda_{1}}+\frac{\varepsilon_{o}}{2} E_{o}^{(\mathrm{o})^{2}}-\frac{1}{2 \mu_{o}} B_{o}^{(\mathrm{o})^{2}}, \quad \hat{t}_{3}=\lambda_{3} \frac{\partial W}{\partial \lambda_{3}}-\lambda_{1} \frac{\partial W}{\partial \lambda_{1}}+\frac{\varepsilon_{o}}{2} E_{o}^{(\mathrm{o})^{2}}-\frac{1}{2 \mu_{o}} B_{o}^{(\mathrm{o})^{2}},
$$

respectively.

\subsubsection{Case $\mathbf{a}_{0}=\mathbf{E}_{3}, \mathbb{E}=E_{o} \mathbf{e}_{3}$ and $\mathbb{B}=B_{o} \mathbf{e}_{2}$}

In this case from (28), (30)-(32) we obtain

$$
\begin{aligned}
& \aleph_{1}=\aleph_{2}=0, \quad \aleph_{3}=1, \quad \beth_{1}=0, \quad \beth_{2}=1, \quad \beth_{3}=0, \quad \beth_{1}=\beth_{2}=0, \quad I_{3}=1, \\
& \alpha_{1}=\alpha_{2}=0, \quad \alpha_{3}=1, \quad \beta_{1}=0, \quad \beta_{2}=1, \quad \beta_{3}=0, \quad \gamma_{1}=\gamma_{2}=0, \quad \gamma_{3}=1, \\
& \zeta_{i}=0, \quad \kappa_{1}=\kappa_{2}=0, \quad \kappa_{3}=1, \quad \varrho_{i}=0, \quad \eta_{i}=0, \quad \chi_{i}=0, \quad \iota_{i}=0 .
\end{aligned}
$$

Eq. (71) is the same and from (39), (43) we have $\frac{\partial W}{\partial \mathbf{b}}=2 \frac{\partial W}{\partial \beta_{2}} \mathbf{E}_{2}$ and $\frac{\partial W}{\partial \mathbf{e}}=2\left(\frac{\partial W}{\partial \gamma_{3}}+\frac{\partial W}{\partial \kappa_{3}}\right) \mathbf{E}_{1}$. Furthermore, from $(33)_{2,3}$, we obtain

$$
\mathbb{H}=\lambda_{2}^{-1} \frac{\partial W}{\partial B_{\mathrm{L}}} \mathbf{e}_{2}, \quad \mathbb{D}=-\lambda_{3} \frac{\partial W}{\partial E_{\mathrm{L}}} \mathbf{e}_{3}
$$


Let us assume that

$$
\mathbb{B}^{(o)}=B_{o}^{(o)} \mathbf{e}_{2}, \quad \mathbb{E}^{(o)}=E_{o} \mathbf{e}_{3},
$$

then $(11)_{1,2}$ are satisfied automatically. On the other hand from (5) we have $\mathbb{H}^{(o)}=\frac{1}{\mu_{o}} B_{o}^{(\circ)} \mathbf{e}_{2}$ and $\mathbb{D}^{(o)}=\varepsilon_{o} E_{o} \mathbf{e}_{3}$, and $(11)_{3}$ is also satisfied automatically. In a similar way, from $(11)_{4}$ we have

$$
\lambda_{2}^{-1} \frac{\partial W}{\partial B_{\mathrm{L}}}=\frac{1}{\mu_{o}} B_{o}^{(\mathrm{o})},
$$

which can be used to find $B_{o}$ as a function of $B_{o}^{(\circ)}$. Using (113) in (13), it can be obtained that $\tau_{\mathrm{M}_{11}}=-\frac{\varepsilon_{o}}{2} E_{o}^{2}-\frac{1}{2 \mu_{o}} B_{o}^{(\mathrm{o})^{2}}$, and from (12), (71) for the surfaces $X_{1}= \pm \frac{L_{1}}{2}$ we obtain $p=\lambda_{1} \frac{\partial W}{\partial \lambda_{1}}+\frac{\varepsilon_{o}}{2} E_{o}^{2}+\frac{1}{2 \mu_{o}} B_{o}^{(\mathrm{o})^{2}}$. Furthermore, from (71) we have

$$
\hat{t}_{2}=\lambda_{2} \frac{\partial W}{\partial \lambda_{2}}-\lambda_{1} \frac{\partial W}{\partial \lambda_{1}}-\frac{\varepsilon_{o}}{2} E_{o}^{2}-\frac{1}{2 \mu_{o}} B_{o}^{(\mathrm{o})^{2}}, \quad \hat{t}_{3}=\lambda_{3} \frac{\partial W}{\partial \lambda_{3}}-\lambda_{1} \frac{\partial W}{\partial \lambda_{1}}-\frac{\varepsilon_{o}}{2} E_{o}^{2}-\frac{1}{2 \mu_{o}} B_{o}^{(\mathrm{o})^{2}} .
$$

For the sake of brevity the following cases are presented in Appendix A: Case $\mathbf{a}_{0}=\mathbf{E}_{2}$, $\mathbb{E}=E_{o} \mathbf{e}_{3}$ and $\mathbb{B}=B_{o} \mathbf{e}_{1}$, Case $\mathbf{a}_{0}=\mathbf{E}_{1}, \mathbb{E}=E_{o} \mathbf{e}_{3}$ and $\mathbb{B}=B_{o} \mathbf{e}_{1}$, and Case $\mathbf{a}_{0}=\mathbf{E}_{3}, \mathbb{E}=E_{o} \mathbf{e}_{3}$ and $\mathbb{B}=B_{o} \mathbf{e}_{1}$.

\subsection{Shear of a slab}

In this problem for the same slab described in Section 4.2, we assume

$$
x_{1}=X_{1}, \quad x_{2}=X_{2}+\kappa X_{3}, \quad x_{3}=X_{3},
$$

where $\kappa$ is a constant. For the electric field and the magnetic induction we consider only one case, wherein

$$
\mathbb{E}_{\mathrm{L}}=E_{\mathrm{L}_{o}} \mathbf{E}_{3}, \quad \mathbb{B}_{\mathrm{L}}=B_{\mathrm{L}_{o}} \mathbf{E}_{3},
$$

where $E_{\mathrm{L}_{o}}$ and $B_{\mathrm{L}_{o}}$ are constants. For the direction $\mathbf{a}_{0}$, three cases are studied, namely

$$
\mathbf{a}_{0}=\mathbf{E}_{3}, \quad \mathbf{a}_{0}=\mathbf{E}_{2}, \quad \mathbf{a}_{0}=c \mathbf{E}_{2}+s \mathbf{E}_{3},
$$

where $c=\cos \xi, s=\sin \xi$, and $\xi$ is the angle between $\mathbf{a}_{0}$ and $X_{2}$. From (116) and (1) we obtain $\mathbf{F}=\sum_{i=1}^{3} \mathbf{e}_{i} \otimes \mathbf{E}_{i}+\kappa \mathbf{e}_{2} \otimes \mathbf{E}_{3}$. Moreover, using $\operatorname{det} \mathbf{F}=1$ and the previous condition, we obtain

$$
\mathbf{C}=\mathbf{E}_{1} \otimes \mathbf{E}_{1}+\mathbf{E}_{2} \otimes \mathbf{E}_{2}+\left(1+\kappa^{2}\right) \mathbf{E}_{3} \otimes \mathbf{E}_{3}+\kappa\left(\mathbf{E}_{2} \otimes \mathbf{E}_{3}+\mathbf{E}_{3} \otimes \mathbf{E}_{2}\right) .
$$

Using the above relations, the following expressions are obtained

$$
\lambda_{1}=1, \quad \lambda_{2}=\frac{1}{\sqrt{2}} \sqrt{2+\kappa^{2}-\kappa \sqrt{4+\kappa^{2}}}, \quad \lambda_{3}=\frac{1}{\sqrt{2}} \sqrt{2+\kappa^{2}+\kappa \sqrt{4+\kappa^{2}}}
$$

and

$$
\mathbf{u}_{1}=\mathbf{E}_{1}, \quad \mathbf{u}_{2}=\frac{1}{U_{2}}\left(\Upsilon_{2} \mathbf{E}_{2}+\mathbf{E}_{3}\right), \quad \mathbf{u}_{3}=\frac{1}{U_{3}}\left(\Upsilon_{3} \mathbf{E}_{2}+\mathbf{E}_{3}\right),
$$

where

$$
\begin{aligned}
& \Upsilon_{2}=-\frac{1}{2}\left(\kappa+\sqrt{4+\kappa^{2}}\right), \quad \Upsilon_{3}=\frac{1}{2}\left(-\kappa+\sqrt{4+\kappa^{2}}\right), \\
& U_{2}=\sqrt{1+\Upsilon_{2}^{2}}, \quad U_{3}=\sqrt{1+\Upsilon_{3}^{2}} .
\end{aligned}
$$


Finally from (117) and (15), (28) we obtain

$$
\mathbb{B}=\kappa B_{\mathrm{L}_{o}} \mathbf{e}_{2}+B_{\mathrm{L}_{o}} \mathbf{e}_{3}, \quad \mathbb{E}=E_{\mathrm{L}_{o}} \mathbf{e}_{3}, \quad \mathbb{b}=\mathbf{E}_{3}, \quad \mathbb{e}=\mathbf{E}_{3}
$$

and $B_{\mathrm{L}}=B_{\mathrm{L}_{o}}, E_{\mathrm{L}}=E_{\mathrm{L}_{o}}$.

\subsubsection{Case $\mathrm{a}_{0}=\mathrm{E}_{3}$}

From (28), (30)-(32) and (121) for the invariants we obtain

$$
\begin{aligned}
& \aleph_{1}=0, \quad \aleph_{2}=\frac{1}{U_{2}}, \quad \aleph_{3}=\frac{1}{U_{3}}, \quad \beth_{1}=0, \quad \beth_{2}=\frac{1}{U_{2}}, \quad \beth_{3}=\frac{1}{U_{3}}, \\
& \beth_{1}=0, \quad \beth_{2}=\frac{1}{U_{2}}, \quad \beth_{3}=\frac{1}{U_{3}}, \quad \alpha_{1}=0, \quad \alpha_{2}=\frac{1}{U_{2}^{2}}, \quad \alpha_{3}=\frac{1}{U_{3}^{2}} \\
& \beta_{1}=0, \quad \beta_{2}=\frac{1}{U_{2}^{2}}, \quad \beta_{3}=\frac{1}{U_{3}^{2}}, \quad \gamma_{1}=0, \quad \gamma_{2}=\frac{1}{U_{2}^{2}}, \quad \gamma_{3}=\frac{1}{U_{3}^{2}} \\
& \zeta_{1}=0, \quad \zeta_{2}=0, \quad \zeta_{3}=\frac{1}{U_{3}}, \quad \kappa_{1}=\kappa_{2}=0, \quad \kappa_{3}=\frac{1}{U_{3}}, \quad \varrho_{1}=\varrho_{2}=0, \quad \varrho_{3}=1, \\
& \eta_{1}=\eta_{2}=0, \quad \eta_{3}=1, \quad \chi_{1}=\chi_{2}=0, \quad \chi_{3}=\frac{1}{U_{3}}, \quad \iota_{1}=\iota_{2}=0, \quad \iota_{3}=\frac{1}{U_{3}} .
\end{aligned}
$$

From (38) we have $\left(\frac{\partial W}{\partial \mathbf{C}}\right)_{12}=0,\left(\frac{\partial W}{\partial \mathbf{C}}\right)_{13}=0$ and

$$
\begin{aligned}
\left(\frac{\partial W}{\partial \mathbf{C}}\right)_{23}= & \frac{1}{\left(\lambda_{2}^{2}-\lambda_{3}^{2}\right)}\left[\left(\frac{\partial W}{\partial \alpha_{2}}-\frac{\partial W}{\partial \alpha_{3}}\right) \frac{1}{U_{2} U_{3}}+\frac{1}{2}\left(\frac{\partial W}{\partial \zeta_{2}}-\frac{\partial W}{\partial \zeta_{3}}\right) \frac{1}{U_{2}}\right. \\
& +\frac{1}{2}\left(\frac{\partial W}{\partial \kappa_{2}}-\frac{\partial W}{\partial \kappa_{3}}\right) \frac{1}{U_{2}}+\frac{1}{2}\left(\frac{\partial W}{\partial \chi_{2}}-\frac{\partial W}{\partial \chi_{3}}\right) \frac{1}{U_{2}} \\
& \left.+\frac{1}{2}\left(\frac{\partial W}{\partial \iota_{2}}-\frac{\partial W}{\partial \iota_{3}}\right) \frac{1}{U_{2}}\right] .
\end{aligned}
$$

Furthermore, for the nonzero components of the total stress from $(33)_{1}$, we have

$$
\begin{aligned}
\tau_{11} & =2 \frac{\partial W}{\partial C_{11}}-p, \quad \tau_{22}=2\left[\frac{\partial W}{\partial C_{22}}+\kappa\left(2 \frac{\partial W}{\partial C_{23}}+\kappa \frac{\partial W}{\partial C_{33}}\right)\right]-p, \\
\tau_{33} & =2 \frac{\partial W}{\partial C_{33}}-p, \quad \tau_{23}=2\left(\frac{\partial W}{\partial C_{23}}+\kappa \frac{\partial W}{\partial C_{33}}\right)
\end{aligned}
$$

where $\frac{\partial W}{\partial C_{i i}}$ can be obtained from (34). From (39) we have

$$
\frac{\partial W}{\partial \mathbf{b}}=\ell_{2} \mathbf{u}_{2}+\ell_{3} \mathbf{u}_{3}+\wp \mathbf{E}_{3}
$$

where

$$
\begin{aligned}
& \ell_{2}=\frac{1}{U_{2}}\left(\frac{\partial W}{\partial \zeta_{2}}+\frac{\partial W}{\partial \iota_{2}}\right) \\
& \ell_{3}=2 \frac{\partial W}{\partial \beta_{3}}+\frac{1}{U_{3}}\left(\frac{\partial W}{\partial \zeta_{3}}+\frac{\partial W}{\partial \iota_{2}}\right)+\frac{\partial W}{\partial \varrho_{3}}+\frac{\partial W}{\partial \eta_{3}} \\
& \wp=\frac{1}{U_{3}}\left(\frac{\partial W}{\partial \zeta_{3}}+2 \frac{\partial W}{\partial \chi_{3}}+\frac{\partial W}{\partial \iota_{3}}\right)+\frac{\partial W}{\partial \varrho_{3}}+\frac{\partial W}{\partial \eta_{3}}
\end{aligned}
$$


Taking into account that $(\mathbf{I}-\boldsymbol{b} \otimes \boldsymbol{b})^{\mathrm{T}}=\mathbf{E}_{1} \otimes \mathbf{E}_{1}+\mathbf{E}_{2} \otimes \mathbf{E}_{2}$, from (36) we obtain

$$
\begin{aligned}
& \leftrightarrow_{\mathrm{L}_{1}}=0, \\
& \mathrm{H}_{\mathrm{L}_{2}}=\left(\frac{\partial W}{\partial \mathbb{B}_{\mathrm{L}}}\right)_{2}=\frac{\partial W}{\partial B_{\mathrm{L}}} \frac{1}{U_{2}^{2}}+\frac{1}{B_{\mathrm{L}}}\left(\frac{\Upsilon_{2} \ell_{2}}{U_{2}}+\frac{\Upsilon_{3} \ell_{3}}{U_{3}}\right) \frac{\Upsilon_{2}}{U_{2}}, \\
& \mathrm{H}_{\mathrm{L}_{3}}=\left(\frac{\partial W}{\partial \mathbb{B}_{\mathrm{L}}}\right)_{3}=\frac{\partial W}{\partial B_{\mathrm{L}}} \frac{1}{U_{3}^{2}}+\frac{1}{B_{\mathrm{L}}}\left(\frac{\Upsilon_{2} \ell_{2}}{U_{2}}+\frac{\Upsilon_{3} \ell_{3}}{U_{3}}\right) \frac{\Upsilon_{3}}{U_{3}} .
\end{aligned}
$$

Furthermore, from $(33)_{2}$ (see (22)) the following relations are obtained

$$
\mathbb{H}=\leftrightarrow_{2} \mathbf{e}_{2}+\leftrightarrow_{3} \mathbf{e}_{3}=\leftrightarrow_{L_{2}} \mathbf{e}_{2}+\left(\kappa \uplus_{L_{2}}+\leftrightarrow_{L_{3}}\right) \mathbf{e}_{3} .
$$

On the other hand, from (43) we obtain

$$
\frac{\partial W}{\partial \mathbb{e}}=\bar{\ell}_{2} \mathbf{u}_{2}+\bar{\ell}_{3} \mathbf{u}_{3}+\wp \mathbf{E}_{3}
$$

where

$$
\begin{aligned}
& \bar{\ell}_{2}=\frac{1}{U_{2}}\left(\frac{\partial W}{\partial \kappa_{2}}+\frac{\partial W}{\partial \chi_{2}}\right) \\
& \bar{\ell}_{3}=2 \frac{\partial W}{\partial \gamma_{3}}+\frac{1}{U_{3}}\left(\frac{\partial W}{\partial \kappa_{3}}+\frac{\partial W}{\partial \chi_{2}}\right)+\frac{\partial W}{\partial \varrho_{3}}+\frac{\partial W}{\partial \eta_{3}}, \\
& \bar{\wp}=\frac{1}{U_{3}}\left(\frac{\partial W}{\partial \kappa_{3}}+2 \frac{\partial W}{\partial \iota_{3}}+\frac{\partial W}{\partial \chi_{3}}\right)+\frac{\partial W}{\partial \varrho_{3}}+\frac{\partial W}{\partial \eta_{3}} .
\end{aligned}
$$

From (37) (see (24)) we obtain

$$
\begin{aligned}
\mathbb{D}_{\mathrm{L}_{1}} & =0 \\
-\mathbb{D}_{\mathrm{L}_{2}} & =\left(\frac{\partial W}{\partial \mathbb{E}_{\mathrm{L}}}\right)_{2}=\frac{\partial W}{\partial E_{\mathrm{L}}} \frac{1}{U_{2}^{2}}+\frac{1}{E_{\mathrm{L}}}\left(\frac{\Upsilon_{2} \bar{\ell}_{2}}{U_{2}}+\frac{\Upsilon_{3} \bar{\ell}_{3}}{U_{3}}\right) \frac{\Upsilon_{2}}{U_{2}}, \\
-\mathbb{D}_{\mathrm{L}_{3}} & =\left(\frac{\partial W}{\partial \mathbb{E}_{\mathrm{L}}}\right)_{3}=\frac{\partial W}{\partial E_{\mathrm{L}}} \frac{1}{U_{3}^{2}}+\frac{1}{E_{\mathrm{L}}}\left(\frac{\Upsilon_{2} \bar{\ell}_{2}}{U_{2}}+\frac{\Upsilon_{3} \bar{\ell}_{3}}{U_{3}}\right) \frac{\Upsilon_{3}}{U_{3}} .
\end{aligned}
$$

Similarly, from $(33)_{3}$ we have

$$
\mathbb{D}=\mathbb{D}_{2} \mathbf{e}_{2}+\mathbb{D}_{3} \mathbf{e}_{3}=\left(\mathbb{D}_{\mathrm{L}_{2}}+\kappa \mathbb{D}_{\mathrm{L}_{3}}\right) \mathbf{e}_{2}+\mathbb{D}_{\mathrm{L}_{3}} \mathbf{e}_{3}
$$

For the electric field and magnetic induction outside the slab it is assumed that

$$
\mathbb{E}^{(\mathrm{o})}=E_{o}^{(\mathrm{o})} \mathbf{e}_{3}, \quad \mathbb{B}^{(\mathrm{o})}=B_{2 o}^{(\mathrm{o})} \mathbf{e}_{2}+B_{3_{o}}^{(\mathrm{o})} \mathbf{e}_{3},
$$

and from (5) we have

$$
\mathbb{D}^{(\mathrm{o})}=\varepsilon_{o} E_{o}^{(\mathrm{o})} \mathbf{e}_{3}, \quad \mathbb{H}^{(\mathrm{o})}=\frac{1}{\mu_{o}}\left(B_{2_{o}}^{(\mathrm{o})} \mathbf{e}_{2}+B_{3_{o}}^{(\mathrm{o})} \mathbf{e}_{3}\right) .
$$

For the surfaces $X_{3}= \pm \frac{L_{3}}{2}$ using $(11)_{1,3,4}$, the continuity conditions are satisfied if

$$
B_{3_{o}}^{(\mathrm{o})}=B_{\mathrm{L}_{o}}, \quad \frac{1}{\mu_{o}} B_{2_{o}}^{(\mathrm{o})}=\mathbb{H}_{2}, \quad \varepsilon_{o} E_{o}^{(\mathrm{o})}=\mathbb{D}_{3},
$$


while $(11)_{2}$ is satisfied automatically. From $(151)_{2,3}$, we can obtain, for example, $E_{o}^{(o)}$ and $B_{2_{o}}^{(o)}$ in terms of $B_{\mathrm{L}_{o}}$ and $E_{\mathrm{L}_{o}}$.

Let us assume that for the surfaces $X_{1}= \pm \frac{L_{1}}{2}$, there exists no external mechanical traction. Hence, using (149) in (13) we have $\tau_{\mathrm{M}_{11}}=-\frac{\varepsilon_{o}}{2} E_{o}^{(\mathrm{o})^{2}}-\frac{1}{2 \mu_{o}}\left(B_{2_{o}}^{(\mathrm{o})^{2}}+B_{\mathrm{L}_{o}}^{2}\right)$ and considering (12) and $(131)_{1}$ we obtain $p=\frac{\partial W}{\partial \lambda_{1}}+\frac{\varepsilon_{o}}{2} E_{o}^{(\mathrm{o})^{2}}+\frac{1}{2 \mu_{o}}\left(B_{2_{o}}^{(\mathrm{o})^{2}}+B_{\mathrm{L}_{o}}^{2}\right)$. For the surfaces $X_{3}= \pm \frac{L_{3}}{2}$, let us assume that the total external load is of the form $\hat{\mathbf{t}}^{(3)}=\hat{t}_{2}^{(3)} \mathbf{e}_{2}+\hat{t}_{3}^{(3)} \mathbf{e}_{3}$. Then from (12), (132) and considering the above expression for $p$, we obtain

$$
\hat{t}_{2}^{(3)}=\tau_{23}, \quad \hat{t}_{3}^{(3)}=\tau_{33}=\frac{1}{\lambda_{3}} \frac{\partial W}{\partial \lambda_{3}}-\frac{\partial W}{\partial \lambda_{1}}-\frac{\varepsilon_{o}}{2} E_{o}^{(\mathrm{o})^{2}}-\frac{1}{2 \mu_{o}}\left(B_{2_{o}}^{(\mathrm{o})^{2}}+B_{\mathrm{L}_{o}}^{2}\right) .
$$

It is necessary to mention that the surfaces $X_{2}= \pm \frac{L_{2}}{2}$ are not in general traction free. The unit normal vector to those surfaces is $\mathbf{n}=\frac{1}{\sqrt{1+\kappa^{2}}} \mathbf{e}_{2}-\frac{\kappa}{\sqrt{1+\kappa^{2}}} \mathbf{e}_{3}$ and assuming that the total external traction is $\hat{\mathbf{t}}^{(2)}=\hat{t}_{2}^{(2)} \mathbf{e}_{2}+\hat{t}_{3}^{(2)} \mathbf{e}_{3}$ from (12), (131) 2 , (132) we obtain

$$
\hat{t}_{2}^{(2)}=\frac{1}{\sqrt{1+\kappa^{2}}}\left(\tau_{22}-\kappa \tau_{23}\right), \quad \hat{t}_{3}^{(2)}=\frac{1}{\sqrt{1+\kappa^{2}}}\left(\tau_{23}-\kappa \tau_{33}\right) .
$$

\subsubsection{Case $\mathrm{a}_{0}=\mathrm{E}_{2}$}

In this case from (125)-(129) we obtain:

$$
\aleph_{1}=0, \quad \aleph_{2}=\frac{\Upsilon_{2}}{U_{2}}, \quad \aleph_{3}=\frac{\Upsilon_{3}}{U_{3}}, \quad \zeta_{i}=0, \quad \kappa_{i}=0, \quad \eta_{i}=0, \quad \chi_{i}=0, \quad \iota_{i}=0,
$$

and the rest of the invariants are the same as in the previous case. On the other hand from (38) we obtain

$$
\left(\frac{\partial W}{\partial \mathbf{C}}\right)_{23}=\frac{1}{\left(\lambda_{2}^{2}-\lambda_{3}^{2}\right)}\left(\frac{\partial W}{\partial \alpha_{2}}-\frac{\partial W}{\partial \alpha_{3}}\right) \frac{\Upsilon_{2} \Upsilon_{3}}{U_{2} U_{3}}
$$

and the rest of the expressions for $\left(\frac{\partial W}{\partial \mathbf{C}}\right)_{i j}$ are indentical as in Section 4.4.1. Similarly, the formulae for $\tau_{i j}$ are the same as in (131) and (132). From (39) we obtain $\frac{\partial W}{\partial \mathbf{b}}=$ $\left(\frac{\partial W}{\partial \zeta_{3}}+\frac{\partial W}{\partial \chi_{3}}+2 \frac{\partial W}{\partial \beta_{3}}+\frac{\partial W}{\partial \varrho_{3}}\right) \frac{\Upsilon_{3}}{U_{3}} \mathbf{E}_{2}+\left[\frac{\partial W}{\partial \varrho_{3}}+\left(2 \frac{\partial W}{\partial \beta_{3}}+\frac{\partial W}{\partial \varrho_{3}}\right) \frac{1}{U_{3}}\right] \mathbf{E}_{3}$. Consequently, from (36) (see (22)) we have

$$
\begin{aligned}
\uplus_{\mathrm{L}_{1}} & =0, \\
\leftrightarrow_{\mathrm{L}_{2}} & =\frac{\partial W}{\partial B_{\mathrm{L}}} \frac{1}{U_{2}^{2}}+\left(\frac{\partial W}{\partial \zeta_{3}}+\frac{\partial W}{\partial \chi_{3}}+2 \frac{\partial W}{\partial \beta_{3}}+\frac{\partial W}{\partial \varrho_{3}}\right) \frac{\Upsilon_{2} \Upsilon_{3}}{U_{2} U_{3}}, \\
\mathbb{L}_{\mathrm{L}_{3}} & =\frac{\partial W}{\partial B_{\mathrm{L}}} \frac{1}{U_{3}^{2}}+\left(\frac{\partial W}{\partial \zeta_{3}}+\frac{\partial W}{\partial \chi_{3}}+2 \frac{\partial W}{\partial \beta_{3}}+\frac{\partial W}{\partial \varrho_{3}}\right) \frac{\Upsilon_{3}^{2}}{U_{3}^{2}} .
\end{aligned}
$$

In the case of (43), we obtain $\frac{\partial W}{\partial \mathbf{e}}=\left(\frac{\partial W}{\partial \kappa_{3}}+\frac{\partial W}{\partial \iota_{3}}+2 \frac{\partial W}{\partial \gamma_{3}}+\frac{\partial W}{\partial \varrho_{3}}\right) \frac{\Upsilon_{3}}{U_{3}} \mathbf{E}_{2}+\left[\frac{\partial W}{\partial \varrho_{3}}+\left(2 \frac{\partial W}{\partial \gamma_{3}}+\frac{\partial W}{\partial \varrho_{3}}\right) \frac{1}{U_{3}}\right] \mathbf{E}_{3}$. Furthermore, due to (37) (see (24)), we have

$$
\begin{aligned}
\mathbb{D}_{\mathrm{L}_{1}} & =0, \\
-\mathbb{D}_{\mathrm{L}_{2}} & =\frac{\partial W}{\partial E_{\mathrm{L}}} \frac{1}{U_{2}^{2}}+\left(\frac{\partial W}{\partial \kappa_{3}}+\frac{\partial W}{\partial \iota_{3}}+2 \frac{\partial W}{\partial \gamma_{3}}+\frac{\partial W}{\partial \varrho_{3}}\right) \frac{\Upsilon_{2} \Upsilon_{3}}{U_{2} U_{3}}, \\
-\mathbb{D}_{\mathrm{L}_{3}} & =\frac{\partial W}{\partial E_{\mathrm{L}}} \frac{1}{U_{3}^{2}}+\left(\frac{\partial W}{\partial \kappa_{3}}+\frac{\partial W}{\partial \iota_{3}}+2 \frac{\partial W}{\partial \gamma_{3}}+\frac{\partial W}{\partial \varrho_{3}}\right) \frac{\Upsilon_{3}^{2}}{U_{3}^{2}} .
\end{aligned}
$$


The expressions for the components of $\mathbb{H}$ and $\mathbb{D}$ in terms of the components of $\boldsymbol{H}_{L}$ and $\mathbb{D}_{\mathrm{L}}$ are the same as in (140), (148), and the analysis of the continuity conditions is identical as in the previous section, therefore, these are not repeated here.

\subsubsection{Case $\mathbf{a}_{0}=c \mathbf{E}_{2}+s \mathbf{E}_{3}$}

In this last case, the expressions for the invariants in (28), (30)-(32) are:

$$
\begin{aligned}
& \aleph_{1}=0, \quad \aleph_{2}=\frac{1}{U_{2}}\left(\Upsilon_{2} c+s\right), \quad \aleph_{3}=\frac{1}{U_{3}}\left(\Upsilon_{3} c+s\right), \quad \beth_{1}=0, \quad \beth_{2}=\frac{1}{U_{2}}, \quad \beth_{3}=\frac{1}{U_{3}}, \\
& \beth_{1}=0, \quad \beth_{2}=\frac{1}{U_{2}}, \quad \beth_{3}=\frac{1}{U_{3}}, \quad \alpha_{1}=0, \quad \alpha_{2}=\frac{1}{U_{2}^{2}}\left(\Upsilon_{2} c+s\right)^{2}, \quad \alpha_{3}=\frac{1}{U_{3}^{2}}\left(\Upsilon_{3} c+s\right)^{2}, \\
& \beta_{1}=0, \quad \beta_{2}=\frac{1}{U_{2}^{2}}, \quad \beta_{3}=\frac{1}{U_{3}^{2}}, \quad \gamma_{1}=0, \quad \gamma_{2}=\frac{1}{U_{2}^{2}}, \quad \gamma_{3}=\frac{1}{U_{3}^{2}}, \\
& \zeta_{1}=0, \quad \zeta_{2}=0, \quad \zeta_{3}=\frac{s}{U_{3}}\left(\Upsilon_{3} c+s\right), \quad \kappa_{1}=\kappa_{2}=0, \quad \kappa_{3}=\frac{s}{U_{3}}\left(\Upsilon_{3} c+s\right), \\
& \varrho_{1}=\varrho_{2}=0, \quad \varrho_{3}=1, \quad \eta_{1}=\eta_{2}=0, \quad \eta_{3}=s^{2}, \quad \chi_{1}=\chi_{2}=0, \quad \chi_{3}=\frac{2}{U_{3}}\left(\Upsilon_{3} c+s\right), \\
& \iota_{1}=\iota_{2}=0, \quad \iota_{3}=\frac{s}{U_{3}}\left(\Upsilon_{3} c+s\right) .
\end{aligned}
$$

For $\left(\frac{\partial W}{\partial \mathbf{C}}\right)_{i j}$ the formulae are the same as in Section 4.4.1 with the exception of (38) that becomes

$$
\begin{aligned}
\left(\frac{\partial W}{\partial \mathbf{C}}\right)_{23}= & {\left[\left(\frac{\partial W}{\partial \alpha_{2}}-\frac{\partial W}{\partial \alpha_{3}}\right) \frac{\left(\Upsilon_{3} c+s\right)}{U_{3}}+\frac{s}{2}\left(\frac{\partial W}{\partial \zeta_{2}}-\frac{\partial W}{\partial \zeta_{3}}+\frac{\partial W}{\partial \kappa_{2}}-\frac{\partial W}{\partial \kappa_{3}}\right.\right.} \\
& \left.\left.+\frac{\partial W}{\partial \chi_{2}}-\frac{\partial W}{\partial \chi_{3}}+\frac{\partial W}{\partial \iota_{2}}-\frac{\partial W}{\partial \iota_{3}}\right)\right] \frac{\left(\Upsilon_{2} c+s\right)}{U_{2}} .
\end{aligned}
$$

From (36) and (22), for the components of $\boldsymbol{H}_{\mathrm{L}}$, we obtain

$$
\mathbb{H}_{\mathrm{L}_{1}}=0, \quad \uplus_{\mathrm{L}_{2}}=\frac{\partial W}{\partial B_{\mathrm{L}}} \frac{1}{U_{2}^{2}}+\frac{\ell_{2} \Upsilon_{2}}{B_{\mathrm{L}} U_{2}}, \quad \uplus_{\mathrm{L}_{3}}=\frac{\partial W}{\partial B_{\mathrm{L}}} \frac{1}{U_{3}^{2}}+\frac{\ell_{2} \Upsilon_{3}}{B_{\mathrm{L}} U_{3}},
$$

where

$$
\begin{array}{r}
\ell_{2}=\left[\frac{\partial W}{\partial \eta_{3}} s+\left(\frac{\partial W}{\partial \zeta_{3}}+\frac{\partial W}{\partial \chi_{3}}\right) \aleph_{3}\right] c+\left(\frac{\partial W}{\partial \zeta_{2}}+\frac{\partial W}{\partial \iota_{2}}\right) s \aleph_{2} \frac{\Upsilon_{2}}{U_{2}}+\left[2 \frac{\partial W}{\partial \beta_{3}}+\frac{\partial W}{\partial \varrho_{3}}\right. \\
\left.+\left(\frac{\partial W}{\partial \zeta_{3}} \aleph_{3}+\frac{\partial W}{\partial \eta_{3}} s+\frac{\partial W}{\partial \iota_{3}} \aleph_{3}\right) s\right] \frac{\Upsilon_{3}}{U_{3}} \\
\ell_{3}=\left[\frac{\partial W}{\partial \eta_{3}} s+\left(\frac{\partial W}{\partial \zeta_{3}}+\frac{\partial W}{\partial \chi_{3}}\right) \aleph_{3}\right] s+\left(\frac{\partial W}{\partial \zeta_{2}}+\frac{\partial W}{\partial \iota_{2}}\right) \frac{s \aleph_{2}}{U_{2}}+\left[2 \frac{\partial W}{\partial \beta_{3}}+\frac{\partial W}{\partial \varrho_{3}}\right. \\
\left.+\left(\frac{\partial W}{\partial \zeta_{3}} \aleph_{3}+\frac{\partial W}{\partial \eta_{3}} s+\frac{\partial W}{\partial \iota_{3}} \aleph_{3}\right) s\right] \frac{1}{U_{3}}+\frac{\partial W}{\partial \varrho_{3}}+\left(\frac{\partial W}{\partial \chi_{3}}+\frac{\partial W}{\partial \iota_{3}}\right) s \aleph_{3} .
\end{array}
$$

Finally for the components of $\mathbb{D}_{\mathrm{L}}$ using (37) and (19), we have

$$
\mathbb{D}_{\mathrm{L}_{1}}=0, \quad-\mathbb{D}_{\mathrm{L}_{2}}=\frac{\partial W}{\partial E_{\mathrm{L}}} \frac{1}{U_{2}^{2}}+\frac{\bar{\ell}_{2} \Upsilon_{2}}{E_{\mathrm{L}} U_{2}}, \quad-\mathbb{D}_{\mathrm{L}_{3}}=\frac{\partial W}{\partial E_{\mathrm{L}}} \frac{1}{U_{3}^{2}}+\frac{\bar{\ell}_{2} \Upsilon_{3}}{E_{\mathrm{L}} U_{3}},
$$


where

$$
\begin{aligned}
& \bar{\ell}_{2}=(\left.\frac{\partial W}{\partial \kappa_{2}} \aleph_{3}+\frac{\partial W}{\partial \eta_{3}} s+\frac{\partial W}{\partial \iota_{3}}\right) c+\left(\frac{\partial W}{\partial \kappa_{2}}+\frac{\partial W}{\partial \chi_{2}}\right) s \aleph_{2} \frac{\Upsilon_{2}}{U_{2}}+\left[2 \frac{\partial W}{\partial \gamma_{3}}+\frac{\partial W}{\partial \varrho_{3}}\right. \\
&\left.+\left(\frac{\partial W}{\partial \eta_{3}} s+\frac{\partial W}{\partial \kappa_{3}} \aleph_{3}+\frac{\partial W}{\partial \chi_{3}} \aleph_{3}\right) s\right] \frac{\Upsilon_{3}}{U_{3}} \\
& \bar{\ell}_{3}=\left(\frac{\partial W}{\partial \kappa_{2}} \aleph_{3}+\frac{\partial W}{\partial \eta_{3}} s+\frac{\partial W}{\partial \iota_{3}}\right) s+\left(\frac{\partial W}{\partial \kappa_{2}}+\frac{\partial W}{\partial \chi_{2}}\right) \frac{s \aleph_{2}}{U_{2}}+\left[2 \frac{\partial W}{\partial \gamma_{3}}+\frac{\partial W}{\partial \varrho_{3}}\right. \\
&\left.+\left(\frac{\partial W}{\partial \eta_{3}} s+\frac{\partial W}{\partial \kappa_{2}} \aleph_{3}+\frac{\partial W}{\partial \chi_{3}} \aleph_{3}\right) s\right] \frac{1}{U_{3}}+\frac{\partial W}{\partial \varrho_{3}}+\left(\frac{\partial W}{\partial \iota_{3}}+\frac{\partial W}{\partial \chi_{3}}\right) s \aleph_{3} .
\end{aligned}
$$

The above expressions can be used to calculate the components of $\mathbb{H}$ and $\mathbb{D}$ from $(15)_{2,4}$. The rest of the analysis on the continuity conditions ((11) and (12)) is the same as presented in Section 4.4.1, hence, it is not repeated here.

\subsection{Cylindrical annulus under inflation and extension}

In this last problem, let us consider a cylindrical annulus described in cylindrical coordinates

$$
R_{\mathrm{i}} \leq R \leq R_{\mathrm{o}}, \quad 0 \leq \Theta \leq 2 \pi, \quad 0 \leq Z \leq L,
$$

where it is assumed that

$$
\mathbf{a}_{0}=\left\{\begin{array}{l}
\mathbf{E}_{R} \\
\mathbf{E}_{\Theta}, \\
\mathbf{E}_{Z}
\end{array}, \quad \mathbb{E}=\left\{\begin{array}{l}
E_{r}(r) \mathbf{e}_{r} \\
E_{z}(r) \mathbf{e}_{z}
\end{array}, \quad \mathbb{B}=\left\{\begin{array}{l}
B_{\theta}(r) \mathbf{e}_{\theta} \\
B_{z}(r) \mathbf{e}_{z}
\end{array} .\right.\right.\right.
$$

There are three possible cases for $\mathbf{a}_{0}$ and two cases for $\mathbb{E}$ and for $\mathbb{B}$. It is assumed that the motion is given by

$$
r=f(R), \quad \theta=\Theta, \quad z=\lambda Z,
$$

where $\lambda>0$ is a constant. The deformation gradient and the right Cauchy-Green tensor are $\mathbf{F}=f^{\prime}(R) \mathbf{e}_{r} \otimes \mathbf{E}_{R}+\frac{f(R)}{R} \mathbf{e}_{\theta} \otimes \mathbf{E}_{\Theta}+\lambda \mathbf{e}_{z} \otimes \mathbf{E}_{Z}, \mathbf{C}=\left[f^{\prime}(R)\right]^{2} \mathbf{E}_{R} \otimes \mathbf{E}_{R}+\frac{[f(R)]^{2}}{R^{2}} \mathbf{E}_{\Theta} \otimes \mathbf{E}_{\Theta}+\lambda^{2} \mathbf{E}_{Z} \otimes \mathbf{E}_{Z}$, respectively. From $\operatorname{det} \mathbf{F}=1$ we obtain

$$
r=f(R)=\sqrt{\frac{1}{\lambda}\left(R^{2}-R_{\mathrm{i}}^{2}\right)+r_{\mathrm{i}}^{2}}
$$

where $r_{\mathrm{i}}$ is the inner radius of the annulus in the current configuration. The principal stretches and directions are

$$
\lambda_{1}=\frac{R}{\lambda r}, \quad \lambda_{2}=\frac{r}{R}, \quad \lambda_{3}=\lambda, \quad \mathbf{u}_{1}=\mathbf{E}_{R}, \quad \mathbf{u}=\mathbf{E}_{\Theta}, \quad \mathbf{u}_{3}=\mathbf{E}_{Z}
$$

\subsubsection{Case $\mathbf{a}_{0}=\mathbf{E}_{R}, \mathbb{E}=E_{r}(r) \mathbf{e}_{r}, \mathbb{B}=B_{\theta}(r) \mathbf{e}_{\theta}$}

In this case from $(15)_{1,3}$ and (26), we have

$$
\mathbb{E}_{\mathrm{L}}=\frac{R E_{r}}{\lambda r} \mathbf{E}_{R}, \quad E_{\mathrm{L}}=\frac{R E_{r}}{\lambda r}, \quad \mathbf{e}=\mathbf{E}_{R}, \quad \mathbb{B}_{\mathrm{L}}=\frac{r B_{\theta}}{R} \mathbf{E}_{\Theta}, \quad B_{\mathrm{L}}=\frac{r B_{\theta}}{R}, \quad \boldsymbol{b}=\mathbf{E}_{\Theta} .
$$


From the equations (28) and (30)-(32) for the invariants, we obtain

$$
\begin{aligned}
& \aleph_{1}=1, \quad \aleph_{2}=\aleph_{3}=0, \quad \beth_{1}=1, \quad \beth_{2}=\beth_{3}=0, \quad \beth_{1}=1, \quad \beth_{2}=\beth_{3}=0, \\
& \alpha_{1}=1, \quad \alpha_{2}=\alpha_{3}=0, \quad \beta_{1}=1, \quad \beta_{2}=\beta_{3}=0, \quad \gamma_{1}=1, \quad \gamma_{2}=\gamma_{3}=0, \\
& \zeta_{i}=0, \quad \kappa_{1}=1, \quad \kappa_{2}=\kappa_{3}=0, \quad \varrho_{i}=0, \quad \eta_{i}=0, \quad \chi_{i}=0, \quad \iota_{i}=0 .
\end{aligned}
$$

From (38) it is possible to show $\left(\frac{\partial W}{\partial \mathbf{C}}\right)_{i j}=0$, therefore, $\tau_{i j}=0, i \neq j$. Moreover, from (34) and $(33)_{1}$, we have

$$
\tau_{r r}=\lambda_{1} \frac{\partial W}{\partial \lambda_{1}}-p, \quad \tau_{\theta \theta}=\lambda_{2} \frac{\partial W}{\partial \lambda_{2}}-p, \quad \tau_{z z}=\lambda_{3} \frac{\partial W}{\partial \lambda_{3}}-p .
$$

Since $\lambda_{i}=\lambda_{i}(R)=\lambda_{i}(r)$, from (10) it is possible to show that $p=p(r)$ and (10) becomes

$$
\frac{\mathrm{d} \tau_{r r}}{\mathrm{~d} r}+\frac{1}{r}\left(\tau_{r r}-\tau_{\theta \theta}\right)=0 .
$$

If we assume that on the inner surface of the tube there is a radial traction $P$ (that incorporates the Maxwell stress), then the solution is

$$
\tau_{r r}(r)=\int_{r_{\mathrm{i}}}^{r} \frac{1}{\xi}\left[\lambda_{2}(\xi) \frac{\partial W}{\partial \lambda_{2}}(\xi)-\lambda_{1}(\xi) \frac{\partial W}{\partial \lambda_{1}}(\xi)\right] \mathrm{d} \xi-P,
$$

where from (178) we have $\lambda_{1}(r)=\frac{\sqrt{\lambda\left(r^{2}-r_{\mathrm{i}}^{2}\right)+R_{\mathrm{i}}^{2}}}{\lambda r}$ and $\lambda_{2}(r)=\frac{r}{\sqrt{\lambda\left(r^{2}-r_{\mathrm{i}}^{2}\right)+R_{\mathrm{i}}^{2}}}$. Let us assume that on the outer surface of the tube there is no mechanical load, then from (12) we obtain $\tau_{r r}\left(r_{\mathrm{o}}\right)=\tau_{\mathrm{M}_{r r}}\left(r_{\mathrm{o}}\right)$. Furthermore, from (186), it can be obtained

$$
\int_{r_{\mathrm{i}}}^{r_{\mathrm{o}}} \frac{1}{\xi}\left[\lambda_{2}(\xi) \frac{\partial W}{\partial \lambda_{2}}(\xi)-\lambda_{1}(\xi) \frac{\partial W}{\partial \lambda_{1}}(\xi)\right] \mathrm{d} \xi-P=\tau_{\mathrm{M}_{r r}}\left(r_{\mathrm{o}}\right)
$$

where $r_{\mathrm{o}}=\sqrt{\frac{1}{\lambda}\left(R_{\mathrm{o}}^{2}-R_{\mathrm{i}}^{2}\right)+r_{\mathrm{i}}^{2}}$. The above equation can be used to find $r_{\mathrm{i}}$.

From (36) and $(33)_{2}$ we have

$$
\mathfrak{H}_{\mathrm{L}_{R}}=\mathfrak{H}_{\mathrm{L}_{Z}}=0, \quad \mathbb{H}_{\mathrm{L}_{\Theta}}=\frac{\partial W}{\partial B_{\mathrm{L}}} \quad \Rightarrow \quad \uplus_{r}=\mathfrak{H}_{z}=0, \quad \mathbb{H}_{\theta}=\lambda_{2}^{-1} \mathfrak{H}_{\mathrm{L}_{\Theta}} .
$$

Then in this case from $(2)_{4}$ considering (3) we obtain

$$
\frac{\mathrm{d}}{\mathrm{d} r}\left(r \uplus_{\theta}\right)=0 .
$$

The solution of the above equation is

$$
\mathrm{H}_{\theta}=\frac{c}{r},
$$

where $c$ is a constant. From (188) and (190), it follows

$$
\frac{\partial W}{\partial B_{\mathrm{L}}}=\frac{c}{R(r)} .
$$

From (37) and $(33)_{3}$, we obtain

$$
-\mathbb{D}_{\mathrm{L}_{R}}=\frac{\partial W}{\partial E_{\mathrm{L}}}, \quad \mathbb{D}_{\mathrm{L}_{\Theta}}=\mathbb{D}_{\mathrm{L}_{Z}}=0 \quad \Rightarrow \quad \mathbb{D}_{r}=-\lambda_{1} \frac{\partial W}{\partial E_{\mathrm{L}}}, \quad \mathbb{D}_{\theta}=\mathbb{D}_{z}=0,
$$


and in this case $(2)_{3}$ considering (4) becomes

$$
\frac{\mathrm{d}}{\mathrm{d} r}\left(r \mathbb{D}_{r}\right)=0
$$

whose solution is

$$
\mathbb{D}_{r}=\frac{\bar{c}}{r}
$$

where $\bar{c}$ is another constant. From (192) and (194), we obtain

$$
\frac{\partial W}{\partial E_{\mathrm{L}}}=-\frac{\bar{c} \lambda}{R(r)} .
$$

Equations (191) and (195) can be used to find $E_{r}(r)$ and $B_{\theta}(r)$ as functions of $r, c$ and $\bar{c}$. In those expressions from $(178)$ we have $R(r)=\sqrt{\lambda\left(r^{2}-r_{\mathrm{i}}^{2}\right)+R_{\mathrm{i}}^{2}}$. If the electric field is produced due to the action of electrodes attached on the inner and outer surfaces of the tube, then the electric potential $\varphi$ can be calculated as $-\left[\varphi\left(r_{\mathrm{o}}\right)-\varphi\left(r_{\mathrm{i}}\right)\right]=-\Delta \varphi=\int_{r_{\mathrm{i}}}^{r_{\mathrm{o}}} E_{r}(\xi) \mathrm{d} \xi$.

Assuming that $L \gg R_{\mathrm{o}}$, the continuity conditions (11) are analyzed only for the surfaces $r=r_{\mathrm{i}}, r=r_{\mathrm{o}}$. Let us assume that

$$
\mathbb{E}^{(\mathrm{o})}=E^{(\mathrm{o})}(r) \mathbf{e}_{r} \quad \Rightarrow \quad \mathbb{D}^{(\mathrm{o})}=\varepsilon_{o} E^{(\mathrm{o})}(r) \mathbf{e}_{r} .
$$

Hence, the Maxwell equation (2) 2 considering (3) becomes

$$
\frac{\mathrm{d}}{\mathrm{d} r}\left(r \mathbb{E}_{r}^{(\mathrm{o})}\right)=0
$$

whose solution is

$$
\mathbb{E}_{r}^{(\mathrm{o})}=\frac{\bar{c}^{(\mathrm{o})}}{r}
$$

where $\bar{c}^{(\mathrm{o})}$ is a constant. There is a constant for $r<r_{\mathrm{i}}$ that can be denoted $\bar{c}_{a}^{(\mathrm{o})}$, and a different constant for $r>r_{\mathrm{o}}$ that is denoted $\bar{c}_{b}^{(\mathrm{o})}$. From (198), the continuity condition $(11)_{2}$ is satisfied automatically, whereas $(11)_{3}$ is satisfied if $\mathbb{D}_{r}\left(r_{\mathrm{i}}\right)=\frac{\bar{c}_{a}^{(o)}}{r_{\mathrm{i}}}$ and $\mathbb{D}_{r}\left(r_{\mathrm{o}}\right)=\frac{\bar{c}_{b}^{(o)}}{r_{\mathrm{o}}}$, which from (192) become

$$
-\lambda_{1}\left(r_{\mathrm{i}}\right) \frac{\partial W}{\partial E_{\mathrm{L}}}\left(r_{\mathrm{i}}\right)=\varepsilon_{o} \frac{\bar{c}_{a}^{(\mathrm{o})}}{r_{\mathrm{i}}}, \quad-\lambda_{1}\left(r_{\mathrm{o}}\right) \frac{\partial W}{\partial E_{\mathrm{L}}}\left(r_{\mathrm{o}}\right)=\varepsilon_{o} \frac{\bar{c}_{b}^{(\mathrm{o})}}{r_{\mathrm{o}}}
$$

respectively. The above equations can be used, for example, to find $\bar{c}_{a}^{(\mathrm{o})}$ and $\bar{c}_{b}^{(\mathrm{o})}$.

For the magnetic induction outside the tube we assume

$$
\mathbb{B}^{(\mathrm{o})}=B_{\theta}^{(\mathrm{o})}(r) \mathbf{e}_{\theta}+B_{z}^{(\mathrm{o})}(r) \mathbf{e}_{z} \quad \Rightarrow \quad \mathbb{H}^{(\mathrm{o})}=\frac{1}{\mu_{o}}\left[B_{\theta}^{(\mathrm{o})}(r) \mathbf{e}_{\theta}+B_{z}^{(\mathrm{o})}(r) \mathbf{e}_{z}\right] .
$$

The Maxwell equation $(2)_{4}$ (see (3)) is satisfied if $B_{z}^{(\mathrm{o})}(r)=B_{z_{o}}^{(\mathrm{o})}$, where $B_{z_{o}}^{(\mathrm{o})}$ is a constant and

$$
\frac{\mathrm{d}}{\mathrm{d} r}\left(\frac{r B_{\theta}^{(\mathrm{o})}(r)}{\mu_{o}}\right)=0,
$$


whose solution is

$$
B_{\theta}^{(\mathrm{o})}(r)=\frac{c^{(\mathrm{o})}}{r} \mu_{o}
$$

Due to $(200)_{2}$, we have $\mathbf{H}^{(\mathrm{o})}=\frac{c^{(\mathrm{o})}}{r} \mathbf{e}_{\theta}+\frac{B_{z o}^{(o)}}{\mu_{o}} \mathbf{e}_{z}$, where the constant $c^{(\mathrm{o})}$ can take a value for $r<r_{\mathrm{i}}$ that is denoted $c_{a}^{(\mathrm{o})}$, and a different value for $r>r_{\mathrm{o}}$ that is denoted $c_{b}^{(\mathrm{o})}$. From (188), $(200)_{1}$ and the previous expression for $\mathbf{H}^{(\mathrm{o})}$ the continuity condition $(11)_{4}$ is satisfied if $B_{z_{o}}^{(\mathrm{o})}=0\left((11)_{1}\right.$ is satisfied automatically), and if $\uplus_{\theta}\left(r_{\mathrm{i}}\right)=\frac{c_{a}^{(\mathrm{o})}}{r_{\mathrm{i}}}$ and $\uplus_{\theta}\left(r_{\mathrm{o}}\right)=\frac{c_{b}^{(\mathrm{o})}}{r_{\mathrm{o}}}$, which from (188) are equivalent to

$$
\lambda_{2}^{-1}\left(r_{\mathrm{i}}\right) \frac{\partial W}{\partial B_{\mathrm{L}}}\left(r_{\mathrm{i}}\right)=\frac{c_{a}^{(\mathrm{o})}}{r_{\mathrm{i}}}, \quad \lambda_{2}^{-1}\left(r_{\mathrm{o}}\right) \frac{\partial W}{\partial B_{\mathrm{L}}}\left(r_{\mathrm{o}}\right)=\frac{c_{b}^{(\mathrm{o})}}{r_{\mathrm{o}}}
$$

which can be used to find, for example, $c_{a}^{(\mathrm{o})}$ and $c_{b}^{(\mathrm{o})}$. (187).

Using $(196)_{1}$ and $(200)_{1}$ in (13) we obtain $\tau_{\mathrm{M}_{r r}}=\frac{\varepsilon_{o} \bar{c}^{(o)^{2}}}{2 r^{2}}-\frac{c^{(o)^{2}}}{2 r^{2}}$, which should be used in

4.5.2 Case $\mathbf{a}_{0}=\mathbf{E}_{R}, \mathbb{E}=E_{r}(r) \mathbf{e}_{r}, \mathbb{B}=B_{z}(r) \mathbf{e}_{z}$

In this problem $(180)_{1,2,3}$ are the same, while

$$
\mathbb{B}_{\mathrm{L}}=\lambda^{-1} B_{z} \mathbf{E}_{Z}, \quad \mathbb{b}=\mathbf{E}_{Z},
$$

and from (28), (30)-(32) the invariants that are different from (181)-(183) are

$$
\begin{aligned}
& \aleph_{1}=\aleph_{2}=0, \quad \aleph_{3}=1, \quad \beth_{1}=\beth_{2}=0, \quad \beth_{3}=1, \\
& \alpha_{1}=\alpha_{2}=0, \quad \alpha_{3}=1, \quad \beta_{1}=\beta_{2}=0, \quad \beta_{3}=1 \text {. }
\end{aligned}
$$

From $(33)_{3}$ and (37), the expressions for the components of the electric displacement are the same as in (192), while from $(33)_{2},(36)$ and (39), we obtain

$$
\uplus_{L_{R}}=\uplus_{\mathrm{L}_{\Theta}}=0, \quad \uplus_{\mathrm{L}_{Z}}=\frac{\partial W}{\partial B_{\mathrm{L}}} \Rightarrow \uplus_{r}=\uplus_{\theta}=0, \quad \uplus_{z}(r)=\lambda_{3}^{-1} \mathbb{H}_{\mathrm{L}_{Z}} .
$$

Using (207) in $(2)_{3}$ (see (3)), we obtain

$$
\frac{\mathrm{d} \mathrm{H}_{z}}{\mathrm{~d} r}=0 \quad \Rightarrow \quad \mathrm{H}_{z}(r)=c,
$$

where $c$ is a constant. From (207) and (208), following expressions can be obtained

$$
\lambda^{-1} \frac{\partial W}{\partial B_{\mathrm{L}}}=c .
$$

Regarding the electric field outside the tube, we have (196), while for the magnetic induction considering (204), (207), we have

$$
\mathbb{B}^{(\mathrm{o})}=B_{\theta}^{(\mathrm{o})}(r) \mathbf{e}_{\theta}+B_{z}^{(\mathrm{o})}(r) \mathbf{e}_{z}, \quad \mathbb{H}^{(\mathrm{o})}=\frac{1}{\mu_{o}}\left[B_{\theta}^{(\mathrm{o})}(r) \mathbf{e}_{\theta}+B_{z}^{(\mathrm{o})}(r) \mathbf{e}_{z}\right],
$$


then $(2)_{4}($ see $(3))$ becomes

$$
\frac{\mathrm{d} B_{z}^{(\mathrm{o})}}{\mathrm{d} r}=0 \quad \Rightarrow \quad B_{z}^{(\mathrm{o})}(r)=B_{z_{o}}^{(\mathrm{o})}, \quad \frac{\mathrm{d}}{\mathrm{d} r}\left(r B_{\theta}^{(\mathrm{o})}\right)=0 \quad \Rightarrow \quad B_{\theta}^{(\mathrm{o})}(r)=\frac{\breve{c}}{r}
$$

where $B_{z_{o}}^{(o)}$ and $\breve{c}$ are constants. Those constants are in general different for $r<r_{\mathrm{i}}$ and $r_{\mathrm{o}}>0$, but we avoid using a different notation for them. The continuity conditions $(11)_{4}$ are satisfied if

$$
c=\frac{B_{z_{o}}^{(o)}}{\mu_{o}}, \quad \breve{c}=0
$$

Finally using $(196)_{1},(210)_{1}$ in (13) we obtain $\tau_{\mathrm{M}_{r r}}=\frac{\varepsilon_{o} \bar{c}^{(\circ)}}{2 r^{2}}-\frac{c^{2}}{2 r^{2}}$, which should be replaced in (187).

\subsubsection{Case $\mathbf{a}_{0}=\mathbf{E}_{R}, \mathbb{E}=E_{z}(r) \mathbf{e}_{z}, \mathbb{B}=B_{\theta}(r) \mathbf{e}_{\theta}$}

In this case the expressions for the magnetic induction are given in $(180)_{4,5,6}$, whereas for the electric field we have

$$
\mathbb{E}_{\mathrm{L}}=\lambda E_{z} \mathbf{E}_{Z}, \quad \mathbb{e}=\mathbf{E}_{Z}
$$

and $(2)_{2}($ see $(3))$ is satisfied if

$$
E_{z}(r)=E_{z_{o}}
$$

where $E_{z_{o}}$ is a constant. In this problem, the invariants from (28), (30)-(32) are

$$
\begin{aligned}
& \beth_{1}=0, \quad \beth_{2}=1, \quad \beth_{3}=0, \quad \beth_{1}=\beth_{2}=0, \quad \beth_{3}=1, \\
& \beta_{1}=0, \quad \beta_{2}=1, \quad \beta_{3}=0, \quad \gamma_{1}=\gamma_{2}=0, \quad \gamma_{3}=0, \\
& \zeta_{i}=0, \quad \kappa_{i}=0, \quad \kappa_{i}=0, \quad \varrho_{i}=0, \quad \eta_{i}=0, \quad \chi_{i}=0, \quad \iota_{i}=0 .
\end{aligned}
$$

From $(33)_{3},(37)$ and $(43)$, we obtain

$$
\mathbb{D}_{\mathrm{L}_{R}}=\mathbb{D}_{\mathrm{L}_{\Theta}}=0, \quad-\mathbb{D}_{\mathrm{L}_{Z}}=\frac{\partial W}{\partial E_{\mathrm{L}}} \quad \Rightarrow \quad \mathbb{D}_{r}=\mathbb{D}_{\theta}=0, \quad \mathbb{D}_{z}=\lambda_{3} \mathbb{D}_{\mathrm{L}_{Z}}=-\lambda \frac{\partial W}{\partial E_{\mathrm{L}}} .
$$

Outside the tube, the expression for $\mathbb{B}^{(0)}$ can be assumed to be the same as (200), and from the continuity conditions $(11)_{4}$, the same restrictions on $\mathbb{B}^{(0)}$ are found. Regarding the electric field outside, we assume

$$
\mathbb{E}^{(\mathrm{o})}=E_{z_{o}} \mathbf{e}_{z} \quad \Rightarrow \quad \mathbb{D}^{(\mathrm{o})}=\varepsilon_{o} E_{z_{o}} \mathbf{e}_{z},
$$

and the continuity condition $(11)_{2,3}$ are satisfied automatically. Finally using $(200)_{1}$ and $(219)_{1}$ in (13), we obtain $\tau_{\mathrm{M}_{r r}}=-\frac{\varepsilon_{o} E_{z_{o}}^{2}}{2}-\frac{c^{2}}{2 r^{2} \mu_{0}}$, which should be replaced in (187).

In the rest of the cases described in (176), many of the different expressions for the electromagnetic variables are the same as in the previous sections. Therefore, for the sake of brevity, we do not repeat such calculations here. The major difference corresponds to the expressions for the invariants (28), (30)-(32), which are presented in Appendix B. 


\section{An example for the total energy function $W$}

It is necessary to remark that there is no enough experimental data available in the literature in order to propose a final expression for the function $W$. The purpose of this article and in particular of the previous section on the boundary value problems, is to have a model that can be used to design some experiments that give results useful for such a fitting. But, with the purpose of facilitating the understanding of the kind of coupling we can observe for this model, in this section we propose a simplified expression for the total energy function $W$, following the work of Shariff [70] for transversely isotropic elastic solids. We propose the specific form (in this section round brackets are reserved for the arguments of functions):

$$
W=W_{\mathrm{T}}+N_{1}+N_{2}-\frac{\epsilon_{0} E_{\mathrm{L}}^{2}}{2} \sum_{i=1}^{3} \frac{\gamma_{i}}{\lambda_{i}^{2}}+\frac{B_{\mathrm{L}}^{2}}{2 \mu_{0}} \sum_{i=1}^{3} \beta_{i} \lambda_{i}^{2},
$$

where

$$
\begin{aligned}
W_{\mathrm{T}} & =\mu_{\mathrm{T}} \sum_{i=1}^{3} r_{1}\left(\lambda_{i}\right)+\mu_{1} \sum_{i=1}^{3} \alpha_{i} r_{2}\left(\lambda_{i}\right)+\frac{\beta}{2}\left[\sum_{i=1}^{3} \alpha_{i} r_{3}\left(\lambda_{i}\right)\right]^{2}, \\
N_{1} & =c_{0}\left(E_{\mathrm{L}}\right) \sum_{i=1}^{3} \gamma_{i} r_{4}\left(\lambda_{i}\right), \quad N_{2}=c_{1}\left(B_{\mathrm{L}}\right) \sum_{i=1}^{3} \beta_{i} r_{5}\left(\lambda_{i}\right),
\end{aligned}
$$

where $\mu_{\mathrm{T}}, \mu_{1}$ and $\beta$ are constants, and in the case of the analysis of a cylinder in tension $\alpha_{i}$, $\beta_{i}, \gamma_{i}, i=1,2,3$ are given in (53). To be consistent with infinitesimal elasticity, the single variable functions $r_{p}, p=1,2, \ldots, 5$ must satisfy

$$
\begin{aligned}
& r_{p}(1)=0, \quad r_{1}^{\prime}(1)=r_{2}^{\prime}(1)=r_{4}^{\prime}(1)=r_{5}^{\prime}(1)=0, \quad r_{3}^{\prime}(1)=1, \\
& r_{1}^{\prime \prime}(1)=r_{2}^{\prime \prime}(1)=r_{4}^{\prime \prime}(1)=r_{5}^{\prime \prime}(1)=2 .
\end{aligned}
$$

From (6), (33) $2,3,(36),(37)$ and (220)-(222) it is clear that

$$
\mathbb{D}=\epsilon_{0} \mathbb{E}-\mathbf{F} \frac{\partial N_{1}}{\partial E_{\mathrm{L}}}, \quad \mathbb{P}=-\mathbf{F} \frac{\partial N_{1}}{\partial E_{\mathrm{L}}}
$$

and

$$
\mathbb{B}=\mu_{0}\left[\boldsymbol{H}-\mathbf{F}^{-\mathrm{T}} \frac{\partial N_{2}}{\partial B_{\mathrm{L}}}\right], \quad \mathbf{M}=-\mathbf{F}^{-\mathrm{T}} \frac{\partial N_{2}}{\partial B_{\mathrm{L}}} .
$$

In vacuum $N_{1}=N_{2}=0$ and we recover the relations

$$
\mathbb{D}^{(\mathbf{o})}=\epsilon_{0} \mathbb{E}^{(\mathbf{o})}, \quad \mathbb{B}^{(\mathbf{o})}=\mu_{0} \mathbb{H}^{(\mathbf{o})} .
$$

In order to obtain the plots presented at the end of this section we use the functions and constants

$$
\begin{aligned}
& r_{1}(x)=r_{2}(x)=r(x)=[\ln (x)]^{2}, \quad r_{3}(x)=\ln (x), \quad r_{4}(x)=r_{5}(x)=[x-1]^{2}, \\
& \beta=0[\mathrm{kPa}], \quad \mu_{T}=\mu_{1}=5[\mathrm{kPa}], \quad c_{0}\left(E_{\mathrm{L}}\right)=0.1 \epsilon_{0} E_{\mathrm{L}}^{2}, \quad c_{1}\left(B_{\mathrm{L}}\right)=\frac{B_{\mathrm{L}}^{2}}{0.01 \mu_{0}}
\end{aligned}
$$


and $\epsilon_{0}=8.85 \times 10^{-12}\left[\mathrm{Fm}^{-1}\right], \mu_{0}=4 \pi \times 10^{-7}[\mathrm{H} / \mathrm{m}]$. From the results shown in Section 4.1 the axial component of the stress for the problem of the extension of a cylinder becomes

$$
\begin{aligned}
\tau_{z z}= & {\left[\mu_{T}+\mu_{1}\right] \lambda_{z} r^{\prime}\left(\lambda_{z}\right)+0.2 \epsilon_{0} E_{0}^{2} \lambda_{z}^{3}\left[\lambda_{z}-1\right]+\frac{2 B_{0}^{2}}{0.01 \mu_{0}}\left[1-\frac{1}{\lambda_{z}}\right] } \\
& -\frac{\mu_{T}}{\sqrt{\lambda_{z}}} r^{\prime}\left(\frac{1}{\sqrt{\lambda_{z}}}\right)+\frac{\epsilon_{0} E_{0}^{2}}{2}+\frac{B_{0}^{2}}{2 \mu_{0}} .
\end{aligned}
$$

In Figs. 1 to 3 results for $\tau_{z z}$ are presented. It is clear from Figs. 1 and 2, for the model given in this section, the application of either the electric field or the the magnetic induction, increases the magnitude axial stress $\tau_{z z}$ at a given axial stretch. The application of both the electric field and the magnetic induction further increases the magnitude of the axial stress as shown in Fig. 3.

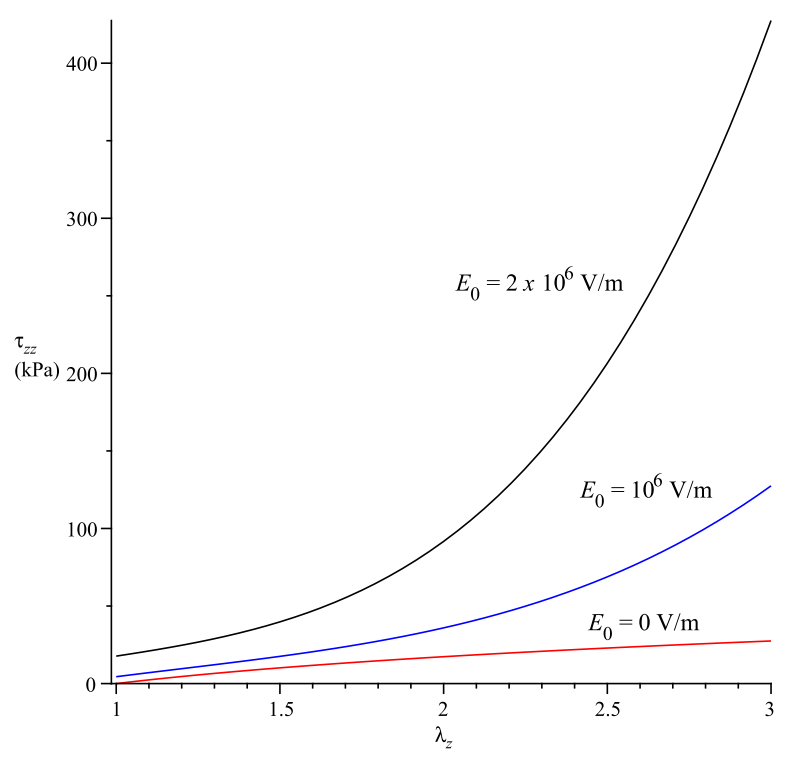

Figure 1: Axial stress $\tau_{z z}$ vs axial stretch $\lambda_{z}$ for various values of the electric field $E_{o}$ when the magnetic induction $B_{o}=0$ [Tesla].

\section{$6 \quad$ Final remarks}

In this article we have presented constitutive equations for nonlinear magneto-electro-elastic bodies, where there are potential applications in the modelling of some types of soft rubberlike materials. One of the novel aspects of this work is the use of spectral invariants for the energy function. Such invariants can facilitate the work of fitting experimental data to find expressions for the energy function. A second contribution of this paper is to provide details of different boundary value problems, which can be used immediately for such experimental research. In a future work after obtaining some expressions for the total energy function from actual experimental data, it is planned to study more boundary value problems, in particular considering inhomogeneous deformations. One limitation of the theory presented here and 


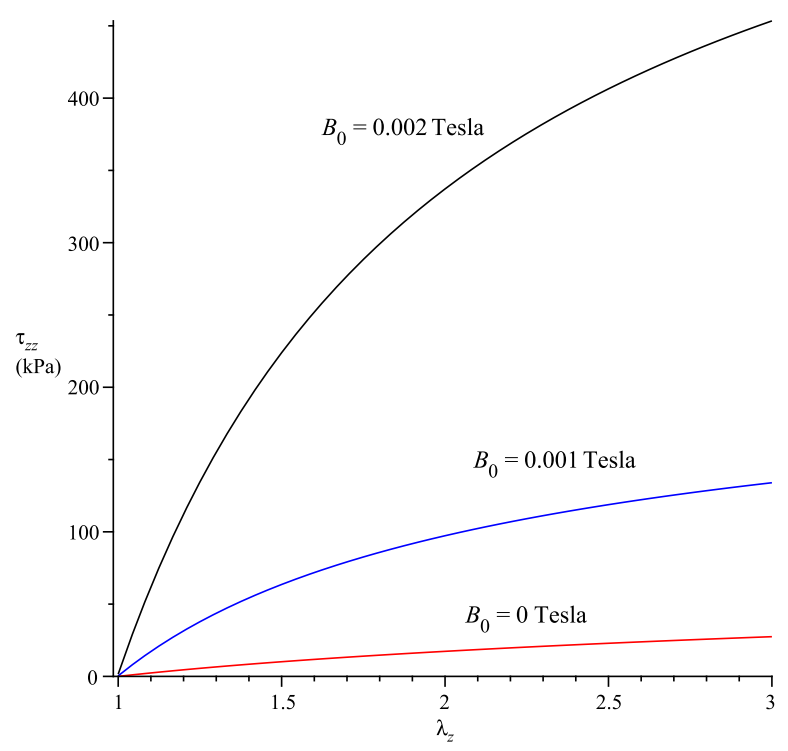

Figure 2: Axial stress $\tau_{z z}$ vs axial stretch $\lambda_{z}$ for various values of the magnetic induction $B_{o}$ when the electric field $E_{o}=0[\mathrm{~V} / \mathrm{m}]$.

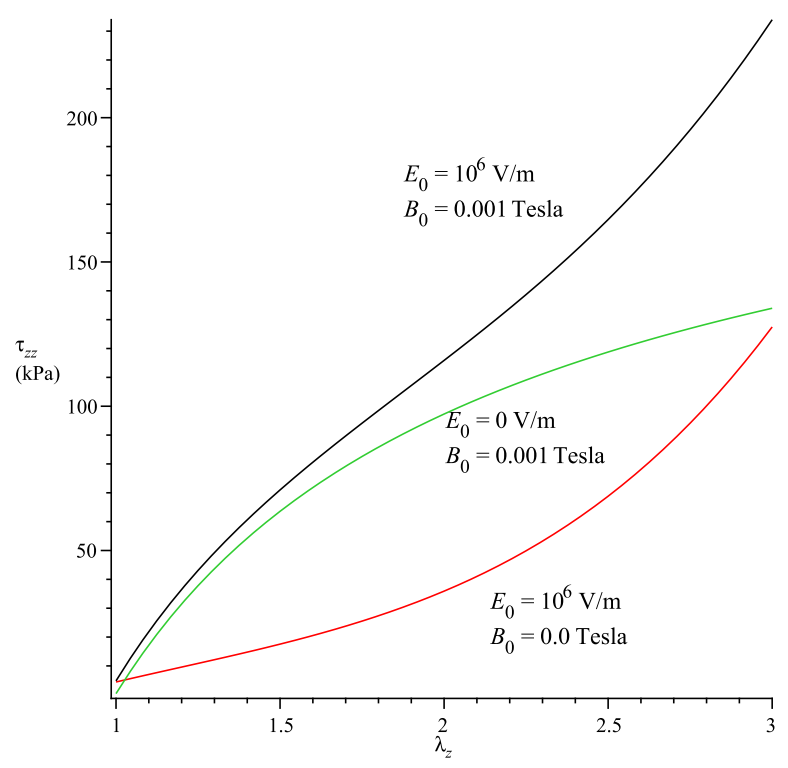

Figure 3: Axial stress $\tau_{z z}$ vs axial stretch $\lambda_{z}$ for various values of the electric field $E_{o}$ and the magnetic induction $B_{o}$.

also in many similar previous works in the literature (see the review in the Introduction), is the assumption of time-independent processes. In a body that can react to electric and magnetic fields moves, there is a coupling between such fields, from where it is necessary to use something called the effective electric field and magnetization (see, for example, $[30,45]$ ). 
Such fully time-dependent model will be analyzed in a future work as well.

\section{Appendix. Additional cases for the boundary value problems}

\section{A. Other cases for the biaxial deformation of a thin plate}

A.1 Case $\mathbf{a}_{0}=\mathbf{E}_{2}, \mathbb{E}=E_{o} \mathbf{e}_{3}$ and $\mathbb{B}=B_{o} \mathbf{e}_{1}$

In this problem from (28), (30)-(32), we have

$$
\begin{array}{llll}
\aleph_{1}=0, & \aleph_{2}=1, \quad \aleph_{3}=0, \quad \beth_{1}=1, \quad \beth_{2}=\beth_{3}=0, \quad \beth_{1}=\beth_{2}=0, & \beth_{3}=1, \\
\alpha_{1}=0, \quad \alpha_{2}=1, \quad \alpha_{3}=0, \quad \beta_{1}=1, \quad \beta_{2}=\beta_{3}=0, & \gamma_{1}=\gamma_{2}=0, & \gamma_{3}=1, \\
\zeta_{i}=0, \quad \kappa_{i}=0, \quad \varrho_{i}=0, \quad \eta_{i}=0, \quad \chi_{i}=0, \quad \iota_{i}=0 . & &
\end{array}
$$

From (39), (5) we obtain $\frac{\partial W}{\partial \mathbf{b}}=2 \frac{\partial W}{\partial \beta_{1}} \mathbf{E}_{1}$ and $\frac{\partial W}{\partial \mathbf{e}}=2 \frac{\partial W}{\partial \gamma_{3}} \mathbf{E}_{3}$. As (71) are the same, repeated calculations are skipped here.

A.2 Case $\mathbf{a}_{0}=\mathbf{E}_{1}, \mathbb{E}=E_{o} \mathbf{e}_{3}$ and $\mathbb{B}=B_{o} \mathbf{e}_{1}$

In this case the expressions, (71) and (89)-(93) are the same. However, the differences appear on the values of some of the invariants, which for this problem from (28), (30)-(32) are:

$$
\begin{aligned}
& \aleph_{1}=\aleph_{2}=0, \quad \aleph_{3}=1, \quad \beth_{1}=1, \quad \beth_{2}=\beth_{3}=0, \quad \beth_{1}=1, \quad \beth_{2}=\beth_{3}=0, \\
& \alpha_{1}=\alpha_{2}=0, \quad \alpha_{3}=1, \quad \beta_{1}=1, \quad \beta_{2}=\beta_{3}=0, \quad \gamma_{1}=1, \quad \gamma_{2}=\gamma_{3}=0, \\
& \zeta_{i}=0, \quad \kappa_{i}=0, \quad \varrho_{1}=1, \quad \varrho_{2}=\varrho_{3}=0, \quad \eta_{i}=0, \quad \chi_{i}=0, \quad \iota_{i}=0 .
\end{aligned}
$$

A.3 Case $\mathbf{a}_{0}=\mathbf{E}_{3}, \mathbb{E}=E_{o} \mathbf{e}_{3}$ and $\mathbb{B}=B_{o} \mathbf{e}_{1}$

In this last case from (28), (30)-(32) we obtain

$$
\begin{aligned}
& \aleph_{1}=\aleph_{2}=0, \quad \aleph_{3}=1, \quad \beth_{1}=1, \quad \beth_{2}=\beth_{3}=0, \quad \beth_{1}=\beth_{2}=0, \quad \beth_{3}=1, \\
& \alpha_{1}=\alpha_{2}=0, \quad \alpha_{3}=1, \quad \beta_{1}=1, \quad \beta_{2}=\beta_{3}=0, \quad \gamma_{1}=\gamma_{2}=0, \quad \gamma_{3}=1, \\
& \zeta_{i}=0, \quad \kappa_{1}=\kappa_{2}=0, \quad \kappa_{3}=1, \quad \varrho_{i}=0, \quad \eta_{i}=0, \quad \chi_{i}=0, \quad \iota_{i}=0 .
\end{aligned}
$$

The rest of the expressions are the same as in Section 4.3.2.

B. Other cases for the problem of the cylindrical annulus. Expressions for the spectral invariants

For the extra cases for the behaviour of the cylindrical annulus the only difference corresponds to the expressions of some of the invariants, which are listed below.

Case $\mathbf{a}_{0}=\mathbf{E}_{R}, \mathbb{E}=E_{z}(r) \mathbf{e}_{z}, \mathbb{B}=B_{z}(r) \mathbf{e}_{z}$ :

$$
\begin{aligned}
& \aleph_{1}=1, \quad \aleph_{2}=\aleph_{3}=0, \quad \beth_{1}=\beth_{2}=0, \quad \beth_{3}=1, \quad \beth_{1}=\beth_{2}=0, \quad \beth_{3}=1, \\
& \alpha_{1}=1, \quad \alpha_{2}=\alpha_{3}=0, \quad \beta_{1}=\beta_{2}=0, \quad \beta_{3}=1, \quad \gamma_{1}=\gamma_{2}=0, \quad \gamma_{3}=1, \\
& \zeta_{i}=0, \quad \kappa_{i}=0, \quad \varrho_{1}=\varrho_{2}=0, \quad \varrho_{3}=1, \quad \eta_{i}=0, \quad \chi_{i}=0, \quad \iota_{i}=0 .
\end{aligned}
$$


Case $\mathbf{a}_{0}=\mathbf{E}_{\Theta}, \mathbb{E}=E_{r}(r) \mathbf{e}_{r}, \mathbb{B}=B_{\theta}(r) \mathbf{e}_{\theta}$ :

$$
\begin{aligned}
& \aleph_{1}=0, \quad \aleph_{2}=1, \quad \aleph_{3}=0, \quad \beth_{1}=0, \quad \beth_{2}=1, \quad \beth_{3}=0, \quad \beth_{1}=1, \quad \beth_{2}=\beth_{3}=0,(243) \\
& \alpha_{1}=0, \quad \alpha_{2}=1, \quad \alpha_{3}=0, \quad \beta_{1}=0, \quad \beta_{2}=1, \quad \beta_{3}=0, \quad \gamma_{1}=1, \quad \gamma_{2}=\gamma_{3}=0,(24) \\
& \zeta_{1}=0, \quad \zeta_{2}=1, \quad \zeta_{3}=0, \quad \kappa_{i}=0, \quad \varrho_{i}=0, \quad \eta_{i}=0, \quad \chi_{i}=0, \quad \iota_{i}=0
\end{aligned}
$$

Case $\mathbf{a}_{0}=\mathbf{E}_{\Theta}, \mathbb{E}=E_{r}(r) \mathbf{e}_{r}, \mathbb{B}=B_{z}(r) \mathbf{e}_{z}$ :

$$
\begin{aligned}
& \aleph_{1}=0, \quad \aleph_{2}=1, \quad \aleph_{3}=0, \quad \beth_{1}=\beth_{2}=0, \quad \beth_{3}=1, \quad \beth_{1}=1, \quad \beth_{2}=\beth_{3}=0, \\
& \alpha_{1}=0, \quad \alpha_{2}=1, \quad \alpha_{3}=0, \quad \beta_{1}=\beta_{2}=0, \quad \beta_{3}=1, \quad \gamma_{1}=1, \quad \gamma_{2}=\gamma_{3}=0, \\
& \zeta_{i}=0, \quad \kappa_{i}=0, \quad \varrho_{i}=0, \quad \eta_{i}=0, \quad \chi_{i}=0, \quad \iota_{i}=0 .
\end{aligned}
$$

Case $\mathbf{a}_{0}=\mathbf{E}_{\Theta}, \mathbb{E}=E_{z}(r) \mathbf{e}_{z}, \mathbb{B}=B_{\theta}(r) \mathbf{e}_{\theta}$ :

$$
\begin{array}{ll}
\aleph_{1}=0, & \aleph_{2}=1, \quad \aleph_{3}=0, \quad \beth_{1}=0, \quad \beth_{2}=1, \quad \beth_{3}=0, \quad \beth_{1}=\beth_{2}=0, \quad \beth_{3}=1,(2 \\
\alpha_{1}=0, \quad \alpha_{2}=1, \quad \alpha_{3}=0, \quad \beta_{1}=0, \quad \beta_{2}=1, \quad \beta_{3}=0, \quad \gamma_{1}=\gamma_{2}=0, & \gamma_{3}=1, \\
\zeta_{1}=0, \quad \zeta_{2}=1, \quad \zeta_{3}=0, \quad \kappa_{i}=0, \quad \varrho_{i}=0, \quad \eta_{i}=0, \quad \chi_{i}=0, \quad \iota_{i}=0
\end{array}
$$

Case $\mathbf{a}_{0}=\mathbf{E}_{\Theta}, \mathbb{E}=E_{z}(r) \mathbf{e}_{z}, \mathbb{B}=B_{z}(r) \mathbf{e}_{z}$ :

$$
\begin{aligned}
& \aleph_{1}=0, \quad \aleph_{2}=1, \quad \aleph_{3}=0, \quad \beth_{1}=\beth_{2}=0, \quad \beth_{3}=1, \quad \beth_{1}=\beth_{2}=0, \quad \beth_{3}=1, \\
& \alpha_{1}=0, \quad \alpha_{2}=1, \quad \alpha_{3}=0, \quad \beta_{1}=\beta_{2}=0, \quad \beta_{3}=1, \quad \gamma_{1}=\gamma_{2}=0, \quad \gamma_{3}=1, \\
& \zeta_{i}=0, \quad \kappa_{i}=0, \quad \varrho_{1}=\varrho_{2}=0, \quad \varrho_{3}=1, \quad \eta_{i}=0, \quad \chi_{i}=0, \quad \iota_{i}=0 .
\end{aligned}
$$

Case $\mathbf{a}_{0}=\mathbf{E}_{Z}, \mathbb{E}=E_{r}(r) \mathbf{e}_{r}, \mathbb{B}=B_{\theta}(r) \mathbf{e}_{\theta}$ :

$$
\begin{aligned}
& \aleph_{1}=\aleph_{2}=0, \quad \aleph_{3}=1, \quad \beth_{1}=0, \quad \beth_{2}=1, \quad \beth_{3}=0, \quad \beth_{1}=1, \quad \beth_{2}=\beth_{3}=0, \\
& \alpha_{1}=\alpha_{2}=0, \quad \alpha_{3}=1, \quad \beta_{1}=0, \quad \beta_{2}=1, \quad \beta_{3}=0, \quad \gamma_{1}=1, \quad \gamma_{2}=\gamma_{3}=0 \text {, } \\
& \zeta_{i}=0, \quad \kappa_{i}=0, \quad \varrho_{i}=0, \quad \eta_{i}=0, \quad \chi_{i}=0, \quad \iota_{i}=0
\end{aligned}
$$

Case $\mathbf{a}_{0}=\mathbf{E}_{Z}, \mathbb{E}=E_{r}(r) \mathbf{e}_{r}, \mathbb{B}=B_{z}(r) \mathbf{e}_{z}$ :

$$
\begin{aligned}
& \aleph_{1}=\aleph_{2}=0, \quad \aleph_{3}=1, \quad \beth_{1}=\beth_{2}=0, \quad \beth_{3}=1, \quad \beth_{1}=1, \quad \beth_{2}=\beth_{3}=0, \\
& \alpha_{1}=\alpha_{2}=0, \quad \alpha_{3}=1, \quad \beta_{1}=\beta_{2}=0, \quad \beta_{3}=1, \quad \gamma_{1}=1, \quad \gamma_{2}=\gamma_{3}=0, \\
& \zeta_{1}=\zeta_{2}=0, \quad \zeta_{3}=1, \quad \kappa_{i}=0, \quad \varrho_{i}=0, \quad \eta_{i}=0, \quad \chi_{i}=0, \quad \iota_{i}=0
\end{aligned}
$$

Case $\mathbf{a}_{0}=\mathbf{E}_{Z}, \mathbb{E}=E_{z}(r) \mathbf{e}_{z}, \mathbb{B}=B_{\theta}(r) \mathbf{e}_{\theta}$ :

$$
\begin{aligned}
& \aleph_{1}=\aleph_{2}=0, \quad \aleph_{3}=1, \quad \beth_{1}=0, \quad \beth_{2}=1, \quad \beth_{3}=0, \quad \beth_{1}=\beth_{2}=0, \quad \beth_{3}=1, \\
& \alpha_{1}=\alpha_{2}=0, \quad \alpha_{3}=1, \quad \beta_{1}=0, \quad \beta_{2}=1, \quad \beta_{3}=0, \quad \gamma_{1}=\gamma_{2}=0, \quad \gamma_{3}=1, \\
& \zeta_{i}=0, \quad \kappa_{1}=\kappa_{2}=0, \quad \kappa_{3}=1, \quad \varrho_{i}=0, \quad \eta_{i}=0, \quad \chi_{i}=0, \quad \iota_{i}=0 .
\end{aligned}
$$

Case $\mathbf{a}_{0}=\mathbf{E}_{Z}, \mathbb{E}=E_{z}(r) \mathbf{e}_{z}, \mathbb{B}=B_{z}(r) \mathbf{e}_{z}$ :

$$
\begin{aligned}
& \aleph_{1}=\aleph_{2}=0, \quad \aleph_{3}=1, \quad \beth_{1}=\beth_{2}=0, \quad \beth_{3}=1, \quad \beth_{1}=\beth_{2}=0, \quad \beth_{3}=1, \\
& \alpha_{1}=\alpha_{2}=0, \quad \alpha_{3}=1, \quad \beta_{1}=\beta_{2}=0, \quad \beta_{3}=1, \quad \gamma_{1}=\gamma_{2}=0, \quad \gamma_{3}=1 \text {, } \\
& \zeta_{1}=\zeta_{2}=0, \quad \zeta_{3}=1, \quad \kappa_{1}=\kappa_{2}=0, \quad \kappa_{3}=1, \quad \varrho_{1}=\varrho_{2}=0, \quad \varrho_{3}=1, \\
& \eta_{1}=\eta_{2}=0, \quad \eta_{3}=1, \quad \chi_{1}=\chi_{2}=0, \quad \chi_{3}=1, \quad \iota_{1}=\iota_{2}=0, \quad \iota_{3}=1 .
\end{aligned}
$$




\section{Acknowledgements:}

The third author would like to extend his sincere appreciation to Santander Staff Mobility fund (2018) that facilitated a short visit to the first author in Departamento de Ingeniería Mecánica, Universidad de Chile, Santiago, Chile.

\section{References}

[1] Amjadi, M., Pichitpajongkit, A., Lee, S., Ryu, S. \& Park, I. (2012). Highly stretchable and sensitive strain sensor based on silver nanowire-elastomer nanocomposite, ACS Nano, $8,5154-5163$.

[2] Barthelemy, M. \& Bibes, A. (2008). Multiferroics: towards a magnetoelectric memory, Nature Materials, 7, 425-426.

[3] Bastola, A. K. \& Hossain, M. (2020). A review on magneto-mechanical characterizations of magnetorheological elastomers, Composites Part B: Engineering, 200, 108348.

[4] Bednarek, S. (1999). The giant magnetostriction in ferromagnetic composites within an elastomer matrix, Applied Physics A, 68, 63-67.

[5] Bellan, C. \& Bossis, G. (2002). Field dependence of viscoelastic properties of MR elastomers, International Jourmal of Modern Physics B, 16, 2447-2453.

[6] Bica, I. (2012). The influence of the magnetic field on the elastic properties of anisotropic magnetorheological elastomers, Journal of Industrial and Engineering Chemistry, 18, $1666-1669$.

[7] Boczkowska, A. \& Awietjan, S. F. (2009). Smart composites of urethane elastomers with carbonyl iron, Journal of Material Science, 44, 4104-4111.

[8] Böse, H. H. (2007). Viscoelastic properties of silicone-based magnetorheological elastomers, International Journal of Modern Physics B, 21, 4790-4797.

[9] Böse, H., Rabindranath, R. \& Ehrlich, J. (2012). Soft magnetorheological elastomers as new actuators for valves, Journal of Intelligent Material Systems and Structures, 23, 989-994.

[10] Brigadnov, I. A. \& A. Dorfmann, (2003). Mathematical modeling of magneto-sensitive elastomers, International Journal of Solids and Structures, 40, 4659-4674.

[11] Brown, W. F. (1966). Magnetoelastic Interactions, Springer, Berlin, 1966

[12] Bustamante, R. (2009). A variational formulation for a boundary value problem considering an electro-sensitive elastomer interacting with two bodies, Mechanics Research Communication, 36, 791-795.

[13] Bustamante, R. (2010). Transversely isotropic nonlinear magneto-active elastomers, Acta Mechanica, 210, 183-214. 
[14] Bustamante, R. (2020). New classes of electro-elastic and thermo-electro-elastic bodies that are not Green elastic. International Journal of Engineering Science, 152, 103308.

[15] Bustamante, R., Dorfmann, A. \& Ogden, R. W. (2008). On variational formulations in nonlinear magnetoelastostatics. Mathematics and Mechanics of Solids, 13, 725-745.

[16] Bustamante, R., Dorfmann, A. \& Ogden, R. W. (2011). Numerical solution of finite geometry boundary value problems in nonlinear magnetoelasticity, International Journal of Solids and Structures, 48, 874-883.

[17] Bustamante, R. \& Rajagopal, K. R. (2013). On a new class of electroelastic bodies: Part I, Proceedings of the Royal Society of London A, 469, 20120521.

[18] Bustamante, R. \& Rajagopal, K. R. (2015). Implicit constitutive relations in nonlinear magneto-elasticity. Proceedings of the Royal Society of London A, 471, 20140959.

[19] Bustamante, R. \& Shariff, M. H. B. M. (2015). A principal axis formulation for nonlinear magnetoelastic deformations: isotropic bodies, European Journal of Mechanics-A/Solids, $50,17-27$.

[20] Bustamante, R. \& Shariff, M. H. B. M. (2016). New sets of spectral invariants for electro-elastic bodies with one and two families of fibres, European Journal of MechanicsA/Solids, 58, 42-53.

[21] Danas, K., Kankanala, S. V. \& Triantafyllidis, N. (2012). Experiments and modelling of iron-particled-filled magnetorheological elastomers, Journal of Mechanics and Physics of Solids, 60, 120-138.

[22] Dang, Z. M., Yuan, J. K., Zha, J. W., Zhou, T., Li, S. T. \& Hu, G. H. (2012). Fundamentals, processes and applications of high-permittivity polymer-matrix composites, Progress in Materials Science, 57, 660-723.

[23] Deng, H. \& Gong, X. (2008). Application of magnetorheological elastomer to vibration absorber. Communication in Nonlinear Science, 13, 1938-1947.

[24] Dorfmann, A. \& Brigdanov, I. A. (2004). Constitutive modelling of magneto-sensitive Cauchy elastic solids, Computational Materials Science, 29, 270-282.

[25] Dorfmann, A. \& Ogden, R. W. (2003). Magnetoelastic modelling of elastomers, European Journal of Mechanics A/Solids, 22, 497-507.

[26] Dorfmann, A. \& Ogden, R. W. (2004). Nonlinear magnetoelastic deformations of elastomers, Acta Mechanica, 167, 13-28.

[27] Dorfmann, A. \& Ogden, R. W. (2004). Nonlinear magnetoelastic deformations, Quarterly Journal of Mechanics and Applied Mathematics, 57, 599-622.

[28] Dorfmann, A. \& Ogden, R. W. (2005). Some problems in nonlinear magnetoelasticity, Zeitschrift für angewandte Mathematik und Physik, 56, 718-745.

[29] Eerenstein, W., Mathur, N. D. \& Scott, J. F. (2006). Multiferroic and magnetoelectric materials. Nature, 442, 759-765. 
[30] Eringen, A. C. \& Maugin, G. A. (1990). Electrodynamics of Continua, Springer-Verlag.

[31] Etier, M., Shvartsman, V. V., Gao, Y., Landers, J., Wende, H. \& Lupascu, D. C. (2013). Magnetoelectric effect in $\left(0_{3}\right)$ CoFe2O4-BaTiO3 (20/80) composite ceramics prepared by the organosol route, Ferroelectrics, 448, 77-85.

[32] Farshad, M. \& Le Roux, M. (2004). A new active noise abatement barrier system. Polymer Testing, 23, 855-860.

[33] Fetisov, Y. K., Bush, A. A., Kamentsev, K. E., Ostashchenko, Y. A. \& Srinivasan, G. (2006). Ferritepiezoelectric multilayers for magnetic field sensors, IEEE. Sensors Journal, 6, 935-938.

[34] Fiebig, M. (2005). Revival of the magnetoelectric effect, Journal of Physics D: Applied Physics, 38, R123.

[35] Garcia-Gonzalez, D. \& Hossain, M. (2020). A microstructural-based approach to model magneto-viscoelastic materials at finite strains, International Journal of Solids and Structure, (In Press) DOI:10.1016/j.ijsolstr.2020.10.028.

[36] Ghafoorianfar, N., Wang, X. \& Gordaninejad, F. (2013). On the sensing of magnetorheological elastomers. Sensors and Smart Structures Technologies for Civil, Mechanical, and Aerospace Systems, Eds. Lynch, JP., Yun, CB., Wang, KW., Proc. of SPIE vol.8692, paper 869214.

[37] Gich, M., Fina, I., Morelli, F., Sánchez, A., Alexe, J., Gázquez, M., Fontcuberta, J. \& Roig, A. (2014). Multiferroic iron oxide thin films at room temperature, Advanced Materials, 26, 4645-4652.

[38] Ginder, J. M., Nichols, M. E., Elie, L. D. \& Tardiff, J. L. (1999). Magnetorheological elastomers: properties and applications, in Wuttig, M.R. (Ed.), SPIE Proceedings 3675, Smart Structures and Materials: Smart Materials Technologies, 131-138. SPIE Press.

[39] Griffiths, D. J. (1998). Introduction to Electrodynamics, 3rd ed. Prentice Hall.

[40] Hossain, M., Saxena, P. \& Steinmann, P. (2015). Modelling the mechanical aspects of the curing process of magneto-sensitive elastomeric materials, International Journal of Solids and Structures, 58, 257-269.

[41] Hossain, M., Chatzigeorgiou, G., Meraghni, F. \& Steinmann, P. (2015). A multi-scale approach to model the curing process in magneto-sensitive polymeric materials, International Journal of Solids and Structures, 69-70, 34-44.

[42] Hu, W., Lum, G. Z., Mastrangeli, M. \& Sitti, M. (2018). Small-scale soft-bodied robot with multimodal locomotion, Nature, 554, 81-85.

[43] Jolly, M. R., Carlson, J. D., \& Muñoz, B. C. (1996). A model of the behaviour of magnetorheological materials, Smart Materials and Structures, 5, 607-614.

[44] Kim, Y., Yuk, H., Zhao, R., Chester, S. A. \& Zhao, X. (2018). Printing ferromagnetic domains for untethered fast-transforming soft materials, Nature, 558, 274-279. 
[45] A. Kovetz, Electromagnetic Theory, University Press, Oxford, 2000

[46] Krichen, S., Liu, L. \& Sharma, P. (2017). Biological cell as a soft magnetoelectric material: elucidating the physical mechanisms underpinning the detection of magnetic fields by animals, Physics Review E, 96, 042404.

[47] Liu, L. \& Sharma, P. (2013). Giant and universal magnetoelectric coupling in soft materials and concomitant ramifications for materials science and biology, Physics Review $E, 88,040601$.

[48] Liu, L. (2014). An energy formulation of continuum magneto-electro-elasticity with applications, Journal of Mechanics and Physics of Solids, 63, 451-480.

[49] Lu, H., Zhang, M., Yang, Y., Huang, Q., Fukuda, T., Wang, Z. \& Shen, Y. (2018). A bioinspired multilegged soft millirobot that functions in both dry and wet conditions, Nature Commications, 9, 3944.

[50] Ma, J., Hu, J., Li, Z. \& Nan, C. W. (2011). Recent progress in multiferroic magnetoelectric composites: from bulk to thin films, Advanced Materials, 23, 1062-1087.

[51] Maugin, G. A. (1988). Continuum Mechanics of Electromagnetic Solids, North Holland, Amsterdam.

[52] Mehnert, M., Hossain, M. \& Steinmann, P. (2016). On nonlinear thermo-electroelasticity, Proceedings of the Royal Society A, 472, 20160170.

[53] Mehnert, M., Hossain, M. \& Steinmann, P. (2017). Towards a thermo-magnetomechanical coupling framework for magneto-rheological elastomers, International Journal of Solids and Structures, 128, 117-132.

[54] Mehnert, M., Hossain, M. \& Steinmann, P. (2018). Numerical modeling of thermoelectro-viscoelasticity with field-dependent material parameters, International Journal of Non-Linear Mechanics, 126, 13-25.

[55] Monk, P. (2003). Finite Element Methods for Maxwell Equations, Oxford University Press, Clarendon.

[56] Nan, C. W., Bichurin, M. I., Dong, S., Viehland, D. \& Srinivasan, G. (2008). Multiferroic magnetoelectric composites: historical perspective, status, and future directions, Journal of Applied Physics, 103, 031101.

[57] Ogden, R. W. (1997). Non-linear Elastic Deformations, Dover, New York.

[58] Pao, Y. H. (1978). Electromagnetic forces in deformable continua, Mechanics Today, (Nemat- Nasser, S., ed.), Oxford: Pergamon Press. 4, 209-306.

[59] Pyatakov, A. P. \& Zvezdin, A. K. (2012). Magnetoelectric and multiferroic media, Physics-Uspekhi, 55, 557.

[60] Rambausek, M. \& Keip, M. A. (2018). Analytical estimation of non-local deformationmediated magneto-electric coupling in soft composites, Proceedings of the Royal Society A, 474, 20170803. 
[61] Santapuri, S., Lowe, R. L., Bechtel, S. E. \& Dapino, M. J. (2013). Thermodynamic modeling of fully coupled finite-deformation thermo-electro-magneto-mechanical behavior for multifunctional applications, International Journal of Engineering Science, 72, 117-139.

[62] Saxena, P., Hossain, M. \& Steinmann, P. (2013). A theory of finite deformation magnetoviscoelasticity, International Journal of Solids and Structures, 50, 3886-3897.

[63] Saxena, P., Hossain, M. \& Steinmann, P. (2014). Nonlinear magneto-viscoelasticity of transversally isotropic magneto-active polymers, Proceeding Royal Society A, 470, 20140082 .

[64] J. F. Scott, Applications of modern ferroelectrics, Science, 315:954-959, 2007

[65] Sfyris, D., Sfyris, G. I. \& Bustamante, R. (2016). Nonlinear electro-magneto-mechanical constitutive modelling of monolayer graphene, Proceedings of The Royal Society A, 472, 20150750 .

[66] Shariff, M. H. B. M. (2008). Nonlinear transversely isotropic elastic solids: An alternative representation, Quarterly Journal of Mechanics and Applied Mathematics, 61, 129-149.

[67] Shariff, M. H. B. M. (2011). Physical invariants for nonlinear orthotropic solids, International Journal of Solids and Structures, 48, 1906-1914.

[68] Shariff, M. H. B. M. (2013). Nonlinear orthotropic elasticity: only six invariants are independent, Journal of Elasticity, 110, 237-241.

[69] Shariff, M. H. B. M. (2015). On the independence of strain invariants of two preferred direction nonlinear elasticity, International Journal of Engineering Science, 97, 18-25.

[70] Shariff, M.H.B.M. (2017). On the spectral constitutive modelling of transversely isotropic soft tissue: Physical invariants, International Journal of Engineering Science, 120, 199 219.

[71] Shariff, M. H. B. M., Bustamante, R., Hossain, M. \& Steinmann, P. (2017). A novel spectral formulation for transversely isotropic magneto-elasticity, Mathematics and Mechanics of Solids, 22, 1158-1176.

[72] Spaldin, N. A. \& Fiebig, M. (2005). The renaissance of magnetoelectric multiferroics. Science, 309, 391-392.

[73] Spencer, A.J.M. (1971). Theory of Invariants, in: Continuum Physics, Vol.I (ed. A.C. Eringen ), Academic Press., 239-353.

[74] Truesdell, C. A. \& Toupin, R. (1960). The classical field theories. In Handbuch der Physik, Vol.III/1. Berlin, Germany: Springer.

[75] Varga, Z., Filipcsei, G. \& Zrínyi, M. (2006). Magnetic field sensitive functional elastomers with tuneable modulus, Polymer, 47, 227-233.

[76] Velev, J. P., Jaswal, S. S. \& Tsymbal, E. Y. (2011). Multi-ferroic and magnetoelectric materials and interfaces, Philosophical Transaction of Royal Society A, 369, 3069-3097.

[77] Vu, D: K., Possart, G. \& Steinmann, P. (2007). Numerical modeling of non-Linear electroelasticity, International Journal for Numerical Methods in Engineering, 70, 685-704. 